Two layers pantographs: A 2D continuum model accounting for the beams' offset and relative rotations as averages in $\mathrm{SO}(3)$ Lie groups

4 authors:

Ivan Giorgio

Università degli Studi dell'Aquila

104 PUBLICATIONS 4,214 CITATIONS

Università Degli Studi Roma Tre

77 PUBLICATIONS 851 CITATIONS 


\title{
Two layers pantographs: a 2D continuum model accounting for the beams' offset and relative rotations as averages in $\mathrm{SO}(3)$ Lie groups
}

\author{
Ivan Giorgio $^{a, b, *}$, Valerio Varano ${ }^{c, b}$, Francesco dell'Isola ${ }^{a, b}$ and Nicola L. Rizzi ${ }^{c, b}$ \\ ${ }^{a}$ Department of Civil, Construction-Architectural and Environmental Engineering (DICEAA), University of L'Aquila, Italy \\ ${ }^{b}$ International Research Center on Mathematics and Mechanics of Complex Systems (M\&MoCS), University of L’Aquila, Italy \\ ${ }^{c}$ Department of Architecture, Università degli studi Roma Tre, Roma, Italy
}

\section{ARTICLE INFO}

\section{Keywords:}

Elastic surface theory

Generalized continuum models

Pantographic structures

\begin{abstract}
A B S TR ACT
In the problem of the synthesis of metamaterials, the pantographic architecture revealed remarkable potentialities. Indeed it allowed for the synthesis of second gradient 2D (nonlinear) continua: i.e. 2D shells whose deformation energy depends also on the second derivatives of displacements in the tangent directions to the reference configuration. Moreover, pantographic architecture seems to be able to produce metamaterials whose macroscopic elongations are large, albeit remaining in the elastic regime. The theoretically shown potentialities have started to become of «practical» interest thanks to a series of experiments, which were made possible by the recent $3 \mathrm{D}$ additive manufacturing. The actual construction of pantographic architecture has been based on the design of two arrays of beams interconnected by small cylinders, whose behavior can be modeled in different ways: if they are very short they can be regarded as clamps, while if they are short enough as elastic (or inelastic for large rotations) cylindrical hinges connecting the beams of different arrays. Otherwise, they must be modeled as elastic (or inelastic) elements allowing for relative rotations and displacements. In this paper, we focus on this particular case and we introduce, after a homogenization based on heuristic arguments, a 2D generalized continuum model whose kinematics is characterized by two placement and rotation fields (one for each array of beams) and whose deformation energy depends on relative displacements and rotations. The offset between the two beams arrays is proven to be an essential tool for defining effective invariant kinematical deformation measures. In facts, one wants to postulate a deformation energy for the introduced 2D generalized continuum which gives predictions in agreement with those given by the more refined 3D model where the pantographic architecture is described with its maximum geometric complexity and where the constituting material is assumed to be modelable as a standard 3D first gradient continuum. In the present paper, in order to arrive at the correct conjecture for the postulated energy, we consider the concept of averages of rotations in $\mathrm{SO}(3)$ Lie group. The used enriched kinematics is seen to be a possible alternative to the adoption of second gradient $2 \mathrm{D}$ models. Some rather surprising deformation processes are studied, where interesting non-symmetric post-buckling phenomena are observed in both the models used. Mentioned post-buckling has been observed experimentally.
\end{abstract}

\section{Introduction}

The technological push towards the use of materials having tailored properties for specific applications originated a renewed interest in theoretical mechanics, a research field that has often been considered exhausted. Further momentum towards the study of interesting novel problems has been impressed by the development of additive manufacturing technology that allows for the construction of material microstructures whose practical realization has been, until recently, merely unimaginable. In facts, one of the most remarkable consequences of the advancements related to additive manufacturing technology consists of the nearly unlimited possibilities that it opens to material scientists in the realization of lower-scale microstructures for materials to be used at another larger scale (see, e.g., dell'Isola and Steigmann (2020); Yildizdag et al. (2019); Barchiesi et al. (2019c); Steigmann et al. (2015); Avella et al. (1998); Rahali et al. (2020); Eremeyev (2019); Niiranen et al. (2019)). An old problem has been revived in this context. The old problem to which we are referring was the so-called synthesis problem faced in the theory of circuital analog computers. In facts, before the prevalence of digital computer, a very important scientific problem with great technological impact

${ }^{*}$ Corresponding author

@ivan.giorgio@univaq.it (I. Giorgio)

ORCID(s): 0000-0002-0044-9188 (I. Giorgio); 0000-0003-0298-6398 (V. Varano); 0000-0002-3607-1113 (F. dell'Isola); 0000-0001-6543-3067 (N.L. Rizzi) 
was the following one: given a certain system of ODEs or PDEs, as starting point of the analysis, one had to find a circuit whose governing equations were exactly the chosen equations (Bush, 1934; Carter and Kron, 1944; Kron, 1945; MacNeal et al., 1951). The found circuit was called the «analog» circuit of the physical problem to be studied by means of the given equations. These dedicated circuits were very effective in solving exactly one specific PDEs or ODEs system, and therefore, only for that problem, they were quick and effective. However they were absolutely useless for another, even slightly different, system of equations. The concept of analog circuit has been recently exploited for designing novel multi-physics metamaterials based on piezoelectric energy transduction (see dell'Isola et al. (2004); Giorgio et al. (2015); Lossouarn et al. (2016) and the references there cited).

In a completely similar way, one can talk about the problem of «synthesis of mechanical metamaterials». A possible formulation of such a problem can be the following: given a specific deformation energy and a specific larger macro length scale one has to find a suitable microstructure, characterized by specific and lower length-scales, such that a material constituted by using the found microstructure, at the chosen macro scale, is governed by the initially chosen deformation energy.

It has to be remarked that with the concept of «microstructure», we include both i) the geometric micro-shape inside which the constituting micro-material is distributed and ii) the stiffnesses distribution inside the micro-shape. Clearly, one should direct his efforts in finding, also for mechanical metamaterials, some general representation theorems, exactly as done in the theory of analog circuits. Indeed, in this last theory, it has been proven that every linear, dissipative n-port circuital element can be synthesized by means of a graph whose branches are constituted by resistors, capacitors, inductors, transformers, and Tellegen's gyrators arranged in the most convenient way. In a sense, this specific representation theorem has reduced the theory of linear dissipative circuits to the study of such elemental components.

Such kind of general representation theorems in the context of the synthesis of mechanical metamaterials are not available yet, albeit some interesting results are given in (Milton and Cherkaev, 1995; Vasquez et al., 2011; Milton, 2013; Milton et al., 2017; Spagnuolo and Scerrato, 2020). However it seems that, in view of the search of such representation results, the pantographic architecture seems to have remarkable potentialities (see, e.g.,Placidi et al. (2016); Barchiesi and Placidi (2017); dell'Isola et al. (2019a,b); Turco (2019)). The partial results which indicate how relevant are said potentialities include those presented in (Barchiesi et al., 2019a; Turco and Barchiesi, 2019; Barchiesi et al., 2020b,a; Greco, 2020; Eremeyev and Turco, 2020; dell'Isola et al., 2017). In facts, by exploiting the pantographic microstructure, it has been possible to synthesize a rather large class of second gradient 2D continua (Germain, 2020) that can be called «second gradient shells». These shells have a deformation energy that depends not only on the second gradient of the transverse displacement field but also on the second gradients of tangential displacement fields (Steigmann and dell'Isola, 2015; Giorgio et al., 2017; Shirani et al., 2019; Abdoul-Anziz and Seppecher, 2018).

It has been observed also that pantographic micro-architecture seems to allow for the synthesis of metamaterials having a very useful peculiarity: their elastic elongation can be very large when compared with standard materials (see for instance dell'Isola et al. (2015); Barchiesi et al. (2020c)). In facts pantographic structures have been introduced in order to synthesize specific second gradient materials, i.e. materials whose deformation energy depends, in a nonnegligible way, on the second gradient of elongation (see Alibert et al. (2003)).

Moreover the theoretical potentialities, which have been shown by means of numerical simulations and mathematical considerations, (see Eremeyev et al. (2018); Turco et al. (2018); Eremeyev and dell'Isola (2020); Andres et al. (2001)) have shown their possible actual technological impact when some specimens could be constructed by using additive manufacturing technology. A series of experiments has been started (Turco et al., 2019, 2016; De Angelo et al., 2019b; Nejadsadeghi et al., 2019; Spagnuolo et al., 2019; dell'Isola et al., 2019c) and the agreement between theoretical predictions and actual experimental evidence seems rather satisfactory (Andreaus et al., 2018; Tran et al., 2020; Laudato et al., 2019; Ciallella, 2020; Spagnuolo et al., 2020). As a bitter criticism was started in the past about the logical and/or physical «impossibility» of the existence of second gradient materials by using «perfect» pivots in the micro-structure it was possible to prove that «purely» second gradient continua may be synthesized (Seppecher et al., 2011). To transform into real materials the ideal synthesis of a pantographic structure, as obtained in aforementioned papers, has not been simple. First of all one had to overcome the technological challenge consisting in constructing, with additive manufacturing, reasonably perfect pivots. The results presented in (dell'Isola et al., 2019a,b; Golaszewski et al., 2019; Spagnuolo et al., 2019) indicate that this challenge can be won.

The simplest actual construction design of pantographic micro-architecture has been obtained by overlapping two distinct arrays of parallel beams placed, in the reference configuration, in two slightly translated planes. To construct in this way a pantographic shell, therefore, an offset between the two beam arrays needs to be introduced. 

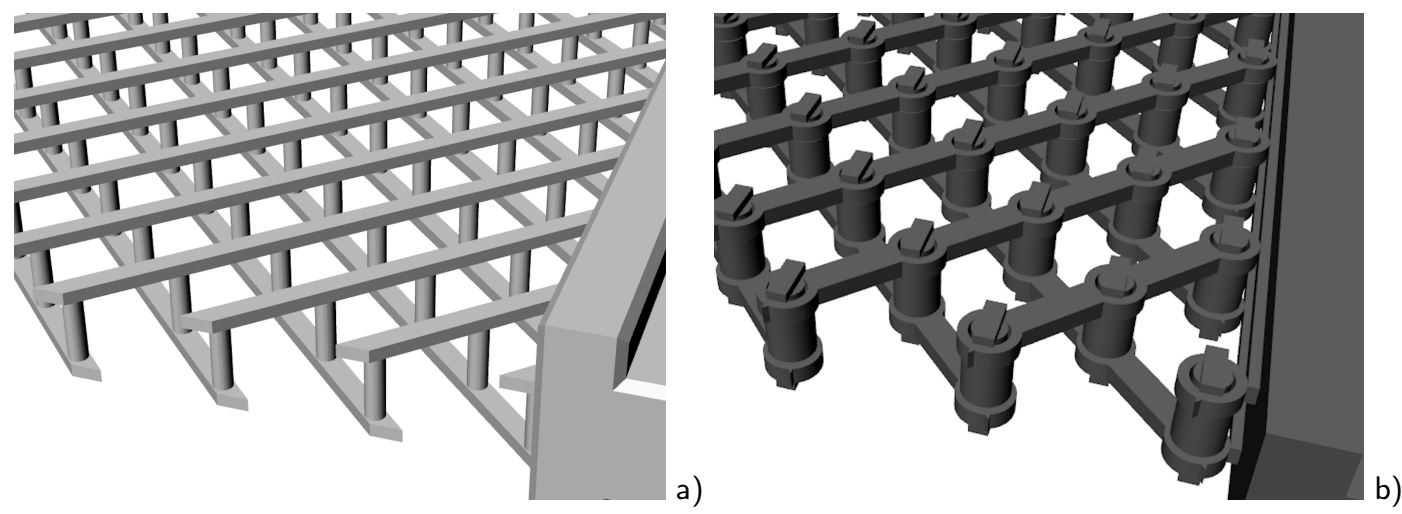

Figure 1: Interconnections between beams of a pantographic sheet: a) deformable cylinders; b) 'perfect' pivots.

As previously anticipated, considered beam arrays are to be somehow interconnected. This can be done either by using small deformable cylinders (see Fig. 1a) or by using some 'perfect pivots' (see Fig. 1b). In the present paper, we focus on the problem of modeling, with models more detailed than second gradient shells, the behavior of those specimens designed with interconnecting cylinders, without resorting to a model where the structural elements of the micro-architecture are modeled as 3D continua. The cylinders mechanical behavior can be modeled in different ways depending on their geometrical characteristics. If their height/diameter ratio is very small, then they must be regarded as clamps, therefore the behavior of the homogenized continuum reduces mainly to a standard first gradient continuum (Pideri and Seppecher, 1997). If instead, their height/diameter ratio is moderately larger, then the interconnecting cylinders can be modeled as elastic (or inelastic in the presence of large rotations) hinges. Finally, when the hight/diameter ratio is large enough then it is more suitable to model them as elastic (or inelastic) elements that allow for relative rotations and displacements of the beams which they are interconnecting.

In this paper, the case of «high» pivotal cylinders is considered in detail. It is proven that, in order to obtain a 2D generalized continuum model that is sufficiently predictive, one can generalize «second gradient shell» models by introducing a more refined kinematics. In this way, we introduce an intermediate complexity model between second gradient shells and aforementioned 3D continua.

In facts, we introduce, after a conjectural homogenization procedure based on heuristic arguments, a 2D continuum model whose richer kinematics is characterized by two placement and rotation fields (one for each array of beams). In other words, we assume that each beam, in the considered arrays, can be modeled as a Timoshenko beam and that the sections interconnected by the cylinders can have a relative displacement and a relative rotation. Moreover we assume that the deformation energy to be postulated for considered 2D generalized continuum depends on each beams' deformation and on the beam-sections relative displacements and rotations. As anticipated, it results that the (variable) offset between the two beams arrays gives a kinematical deformation measure capable to describe pantographic shells deformation patterns.

In facts, one could also model the considered pantographic shell by introducing a more detailed 3D model where its complex micro-geometry is fully taken into account and the material constituting it is modeled as a first gradient $3 \mathrm{D}$ continuum. The drawback of this modeling choice is clear: with the presently available computational tools one needs several millions of finite elements (and too many hours of computing time) before solving a single deformation problem. Moreover, one may have some difficulties in extracting out of the huge amount of information so obtained the synthetic ones that are required to characterize the behavior of conceived metamaterial. Such a more refined modeling procedure has been used, in the present paper, to obtain a numerical identification of the material parameters to be used in the independently postulated reduced order 2D generalized continuum. In facts, by using this 3D refined model one has confirmed the conjecture that was the starting point in the modeling being at the base of the present paper: the performed numerical simulations indicate that the beams arrays offset may play a crucial role in the deformation pattern of the whole pantographic shell.

The main result presented in this paper concerns the determination of the form for the deformation energy to be postulated for introduced 2D generalized continuum that allows for predictions in good agreement with the predictions obtained by using the more refined 3D model. The crucial idea needed to obtain the correct conjecture for the postulated 
energy involves the concept of averages of rotations in SO(3) Lie group (see Moakher (2002)).

We verify that the heuristic identification procedure between the introduced $3 \mathrm{D}$ refined model and the $2 \mathrm{D}$ generalized continuum model (see for more details about identification procedure Placidi et al. (2015); Rosi et al. (2018); Misra and Poorsolhjouy (2015); Yang et al. (2019); Abali et al. (2016); Harrison et al. (2018); Berkache et al. (2019); Rahali et al. (2016)) based on Saint-Venant stiffnesses identification for Timoshenko beams produces a satisfactory agreement between the two models. The obtained identification indicates that the conjectured enriched kinematics is suitable to capture some of the most important deformation features of pantographic shells when using a $2 \mathrm{D}$ generalized continuum model. In facts, some rather surprising deformation processes have been found where very interesting and unexpected non-symmetric post-buckling equilibrium paths are exhibited. These equilibrium paths are observed, with remarkable qualitative and quantitative agreement, in both used 2D and 3D models. Moreover after having calculated them based on the used models, we could find in the literature some experimental evidence which can be consequently fully explained now (Ganzosch et al., 2018; Barchiesi et al., 2019b). In fact, Fig. 11 in (Ganzosch et al., 2018) shows that the out-of-plane displacement field in the shear test fails to be skew symmetric following the same pattern displayed in Fig. 13 of the present paper.

\section{Kinematics of a pantographic structure}

\subsection{Kinematical description of the fibers}

Consider the structure shown in Figure 2 made up by beams regularly distributed on two layers and connected by elements that we call pivots. Each beam has rectangular (rigid) sections while the pivots are small cylinders.

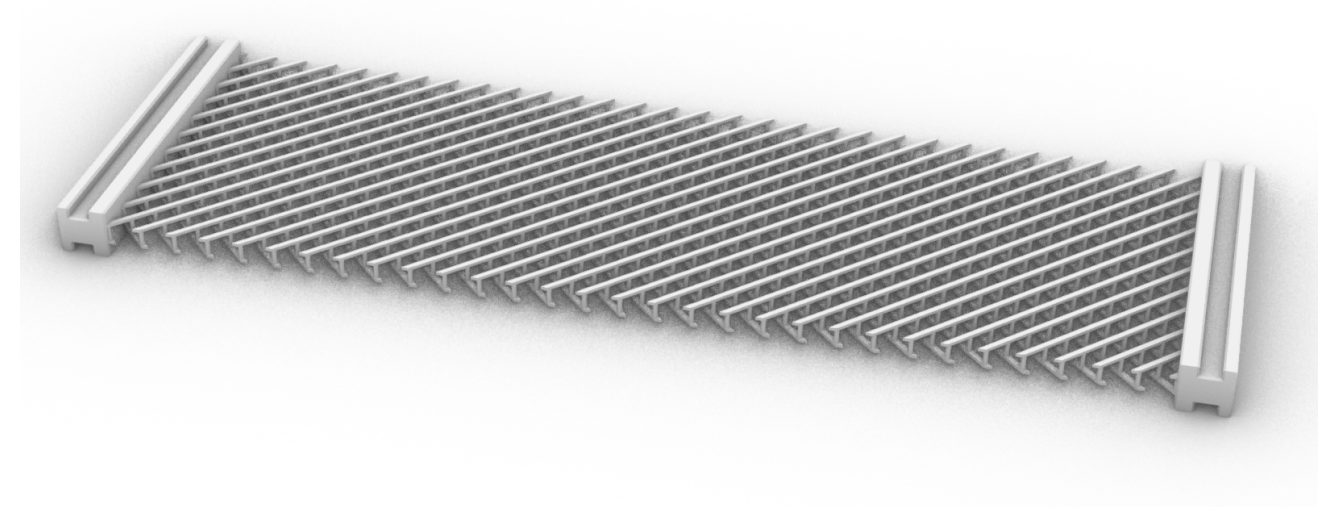

Figure 2: A sample of pantographic structure.

The axes of two prototype beams, one for each layer, in the reference configuration, are shown in Figure 3 where the layers are denoted by the labels 1 and 2 . Besides, with $s_{1}$ and $s_{2}$ we will denote the abscissas along the axes 1 and 2 , respectively.

The kinematics of the beams will be described by the fields

$$
\begin{array}{ll}
{ }^{1} \boldsymbol{u}\left(s_{1}\right), & { }^{1} \boldsymbol{R}\left(s_{1}\right), \\
{ }^{2} \boldsymbol{u}\left(s_{2}\right), & { }^{2} \boldsymbol{R}\left(s_{2}\right),
\end{array}
$$

$\boldsymbol{u}$ being a vector describing the displacement of the points laying on the beam axis and $\boldsymbol{R}$ an orthogonal tensor describing the rotation of the beam sections.

The left superscripts will denote, from now on, that the quantities are referred to the beams lying on the sets 1 and 2 , respectively.

With reference to the orthonormal bases $\boldsymbol{b}_{i}$ and $\boldsymbol{c}_{i}$ shown in Figure 3, the fields in (1) can be given the following component form 


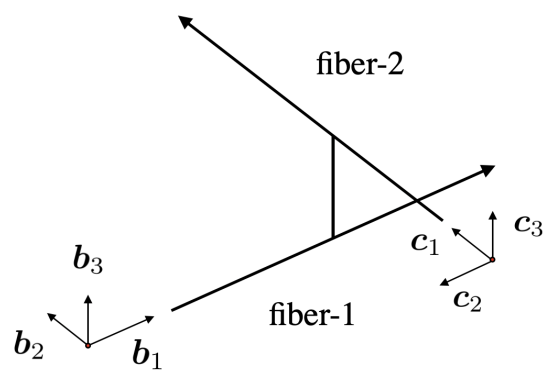

Figure 3: A detail of the pantographic geometry and reference frames.

$$
\begin{aligned}
{ }^{1} u & ={ }^{1} u_{i} b_{i} & { }^{2} u & ={ }^{2} u_{i} b_{i} \\
{ }^{1} \boldsymbol{R} & ={ }^{1} \boldsymbol{R}_{i j} \boldsymbol{b}_{i} \otimes \boldsymbol{b}_{j} & { }^{2} \boldsymbol{R} & ={ }^{2} \tilde{R}_{i j} c_{i} \otimes c_{j}={ }^{2} \boldsymbol{R}_{i j} \boldsymbol{b}_{i} \otimes \boldsymbol{b}_{j} .
\end{aligned}
$$

A useful representation of the components ${ }^{1} R_{i j}$ and ${ }^{2} \tilde{R}_{i j}$ is given in terms of the Bryant angles $\left\{{ }^{1} \varphi_{1},{ }^{1} \varphi_{2},{ }^{1} \varphi_{3}\right\}$ and $\left\{{ }^{2} \varphi_{1},{ }^{2} \varphi_{2},{ }^{2} \varphi_{3}\right\}$, respectively.

The deformation measures for each beam are assumed to be

$$
\begin{aligned}
{ }^{\alpha} \boldsymbol{e} & ={ }^{\alpha} \boldsymbol{R}^{\top}\left({ }^{\alpha} \boldsymbol{X}_{, \alpha}+{ }^{\alpha} \boldsymbol{u}_{, \alpha}\right)-{ }^{\alpha} \boldsymbol{X}_{, \alpha} \\
{ }^{\alpha} \boldsymbol{\kappa} & =\left({ }^{\alpha} \boldsymbol{R}^{\top \alpha} \boldsymbol{R}_{, \alpha}\right)_{\times} \quad(\alpha=1,2),
\end{aligned}
$$

where $\boldsymbol{X}$ denotes the position of a point lying on the axis in the reference configuration, the index after the comma is used to denote differentiation with respect to the abscissa along the beam axis and ${ }^{\alpha} \boldsymbol{K}$ is the axial vector of the skew tensor ${ }^{\alpha} \boldsymbol{R}^{\top \alpha} \boldsymbol{R}_{, \alpha}{ }^{1}$.

\subsection{Kinematical description of the pivots}

As written in Section 2.1 the pivots are cylinders and we will denote by $r$ and $h$ their radius and height, respectively.

We assume that the kinematics (and the mechanical behavior) of a pivot can be described by the sole motion of its bases that, in turn, are assumed to be rigid (circles).

Now let us denote by $\boldsymbol{X}_{1}, \boldsymbol{X}_{2}$, the position of two points belonging to the bases 1 and 2, respectively, in the reference configuration.

In this way, a deformation of the pivot will be described by the transplacement, i.e. the change of the space placement, of its bases by means of the quantities

$$
\begin{array}{lrr}
\boldsymbol{x}_{1} & \text { or } & \boldsymbol{u}_{1}\left(X_{1}\right)=x_{1}-X_{1} \\
\boldsymbol{x}_{2} & \text { or } & \boldsymbol{u}_{2}\left(X_{2}\right)=\boldsymbol{x}_{2}-\boldsymbol{X}_{2} \\
\boldsymbol{R}_{1}, & \boldsymbol{R}_{2} &
\end{array}
$$

where $\boldsymbol{x}_{1}, \boldsymbol{x}_{2}$, are the new positions of $\boldsymbol{X}_{1}, \boldsymbol{X}_{2}$, the vectors $\boldsymbol{u}_{1}, \boldsymbol{u}_{2}$, their displacements, and $\boldsymbol{R}_{1}, \boldsymbol{R}_{2}$, the orthogonal tensors that give the rotations of the bases 1,2 , respectively.

If the pivot undergoes a rigid transplacement we have

$$
\begin{aligned}
& \boldsymbol{R}_{1}=\boldsymbol{R}_{2}=\boldsymbol{R} \\
& \boldsymbol{x}_{2}=\boldsymbol{x}_{1}+\boldsymbol{R}\left(X_{2}-X_{1}\right)
\end{aligned}
$$

\footnotetext{
${ }^{1}$ Given a skew tensor $\boldsymbol{W}$, we denote by $\boldsymbol{W}_{\times}$the axial vector satisfying the identity $\boldsymbol{W} \boldsymbol{u}=\boldsymbol{W}_{\times} \times \boldsymbol{u}, \forall \boldsymbol{u}$.
} 


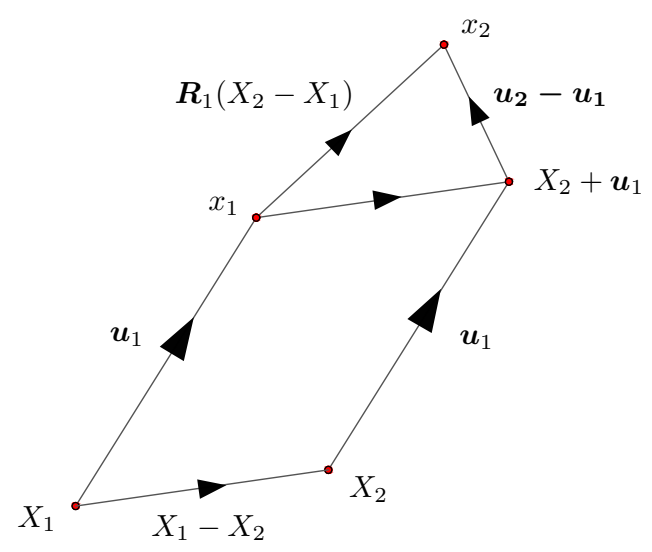

Figure 4: Rigid Transplacement.

while, for a regular transplacement we put

$$
\begin{aligned}
& \boldsymbol{R}_{1} \neq \boldsymbol{R}_{2} \\
& \boldsymbol{x}_{2}=\boldsymbol{x}_{1}+\boldsymbol{R}\left(\boldsymbol{X}_{2}-\boldsymbol{X}_{1}\right)+\boldsymbol{e}
\end{aligned}
$$

where $\boldsymbol{R}$ is any rotation and $\boldsymbol{e}$ is a deformation.

\section{Change in observer: invariance}

Given a reference frame let us denote by $\boldsymbol{X}, \boldsymbol{x}$ the position of a point in the reference and deformed configurations, viewed in that frame, respectively.

Given a fixed point whose position in the reference frame is $\mathcal{O}$ and an orthogonal tensor $\boldsymbol{Q}$, a change in observer applied to the deformed configuration, is defined by the following transformation

$$
x^{*}=\mathcal{O}^{*}+Q(x-\mathcal{O})
$$

where $\mathcal{O}^{*}$ and $\boldsymbol{x}^{*}$ are the positions in the new frame of the point whose positions in the reference frame were $\boldsymbol{x}, \mathcal{O}$, respectively.

In this way the points in the deformed configuration that occupied the positions $\boldsymbol{x}_{1}, \boldsymbol{x}_{2}$ as observed from the reference frame will occupy the positions

$$
\begin{aligned}
& x_{1}^{*}=\mathcal{O}^{*}+Q\left(x_{1}-\mathcal{O}\right) \\
& x_{2}^{*}=\mathcal{O}^{*}+Q\left(x_{2}-\mathcal{O}\right)
\end{aligned}
$$

as observed by the new frame.

Besides a displacement vector changes in the following way

$$
u^{*}=x^{*}-X^{*}=Q(x-X)=Q u \text {. }
$$

Now we state that the strain energy density of the pivot in the deformed configuration is a function of the shape identifiers $\boldsymbol{x}_{1}, \boldsymbol{x}_{2}, \boldsymbol{R}_{1}, \boldsymbol{R}_{2}$, that is

$$
\mathcal{W}=\mathcal{W}\left(x_{1}, x_{1}+\boldsymbol{R}\left(X_{2}-X_{1}\right)+e, \boldsymbol{R}_{1}, \boldsymbol{R}_{2}\right)
$$


where (8) has been used.

We then require that the strain energy density be frame indifferent, that is

$$
\mathcal{W}\left(x_{1}, x_{2}, \boldsymbol{R}_{1}, \boldsymbol{R}_{2}\right)=\mathcal{W}\left(x_{1}^{*}, x_{1}^{*}+\left[\boldsymbol{R}\left(\boldsymbol{X}_{2}-\boldsymbol{X}_{1}\right)\right]^{*}+e^{*}, \boldsymbol{R}_{1}^{*}, \boldsymbol{R}_{2}^{*}\right) .
$$

\subsection{First condition}

Making the following assumptions

$$
\begin{aligned}
\mathcal{O}^{*}= & \mathcal{O}-\left(x_{1}-\mathcal{O}\right) \\
\boldsymbol{Q}= & \boldsymbol{I} \\
& \Rightarrow x_{1}^{*}=\mathcal{O}
\end{aligned}
$$

from (14), (13) we obtain

$$
\begin{aligned}
\mathcal{W}\left(x_{1}, x_{2}, \boldsymbol{R}_{1}, \boldsymbol{R}_{2}\right) & =\mathcal{W}\left(\mathcal{O}, \mathcal{O}+\boldsymbol{R}\left(\boldsymbol{X}_{2}-\boldsymbol{X}_{1}\right)+\boldsymbol{e}, \boldsymbol{R}_{1}, \boldsymbol{R}_{2}\right) \\
& =\mathcal{W}\left(\mathcal{O}+\boldsymbol{R}\left(\boldsymbol{X}_{2}-\boldsymbol{X}_{1}\right)+\boldsymbol{e}, \boldsymbol{R}_{1}, \boldsymbol{R}_{2}\right)
\end{aligned}
$$

that is, $\mathcal{W}$ can not depend on $x_{1}$.

\subsection{Second condition}

Besides, if we assume

$$
\begin{aligned}
\mathcal{O}^{*} & =\mathcal{O}-\left(\boldsymbol{X}_{2}-\boldsymbol{X}_{1}\right) \\
\boldsymbol{Q} & =\boldsymbol{R}^{\top}
\end{aligned}
$$

we obtain

$$
\begin{aligned}
& \mathcal{W}\left(\mathcal{O}^{*}+\left[\boldsymbol{R}\left(\boldsymbol{X}_{2}-\boldsymbol{X}_{1}\right)\right]^{*}+\boldsymbol{e}^{*}, \boldsymbol{R}_{1}^{*}, \boldsymbol{R}_{2}^{*}\right) \\
&=\mathcal{W}\left(\mathcal{O}-\left(\boldsymbol{X}_{2}-\boldsymbol{X}_{1}\right)+\right. \\
&\left.\boldsymbol{R}^{\top}\left[\boldsymbol{R}\left(\boldsymbol{X}_{2}-\boldsymbol{X}_{1}\right)\right]+\boldsymbol{R}^{\top} \boldsymbol{e}, \boldsymbol{R}^{\top} \boldsymbol{R}_{1}, \boldsymbol{R}^{\top} \boldsymbol{R}_{2}\right) \\
&= \mathcal{W}\left(\mathcal{O}+\boldsymbol{R}^{\top} \boldsymbol{e}, \boldsymbol{R}^{\top} \boldsymbol{R}_{1}, \boldsymbol{R}^{\top} \boldsymbol{R}_{2}\right) \\
&= \mathcal{W}\left(\boldsymbol{R}^{\top} \boldsymbol{e}, \boldsymbol{R}^{\top} \boldsymbol{R}_{1}, \boldsymbol{R}^{\top} \boldsymbol{R}_{2}\right)
\end{aligned}
$$

Finally, if we choose $\boldsymbol{R}=\boldsymbol{R}_{1}$, (17) becomes

$$
\mathcal{W}\left(\boldsymbol{R}_{1}^{\top} \boldsymbol{e}, \boldsymbol{I}, \boldsymbol{R}_{1}^{\top} \boldsymbol{R}_{2}\right)=\mathcal{W}\left(\boldsymbol{R}_{1}^{\top} \boldsymbol{e}, \boldsymbol{R}_{1}^{\top} \boldsymbol{R}_{2}\right)
$$

Looking to (17) and (18), we can choose the deformation measures of the pivot depend on $\boldsymbol{R}^{\top} \boldsymbol{e}$ and $\boldsymbol{R}_{1}^{\top} \boldsymbol{R}_{2}, \boldsymbol{R}$ being any orthogonal tensor.

Now, if we label with the $1 p$ and $2 p$ upper left superscripts quantities related to the bases of the pivot connected to the beams lying on the sets 1 and 2, respectively, see Figure 5, eqn. (19), that describe a pivot's deformation, becomes

$$
\begin{array}{lrrr}
{ }^{1 p} \boldsymbol{x} & \text { or } & { }^{1 p} \boldsymbol{u}\left({ }^{1 p} \boldsymbol{X}\right)={ }^{1 p} \boldsymbol{x}-{ }^{1 p} \boldsymbol{X} \\
{ }^{2 p} \boldsymbol{x} & \text { or } & { }^{2 p} \boldsymbol{u}\left({ }^{2 p} \boldsymbol{X}\right)={ }^{2 p} \boldsymbol{X}-{ }^{2 p} \boldsymbol{X} \\
{ }^{1 p} \boldsymbol{R},{ }^{2 p} \boldsymbol{R} &
\end{array}
$$

where ${ }^{1 p} \boldsymbol{X},{ }^{2 p} \boldsymbol{X}$ stand for the reference position of the bases' centers. 


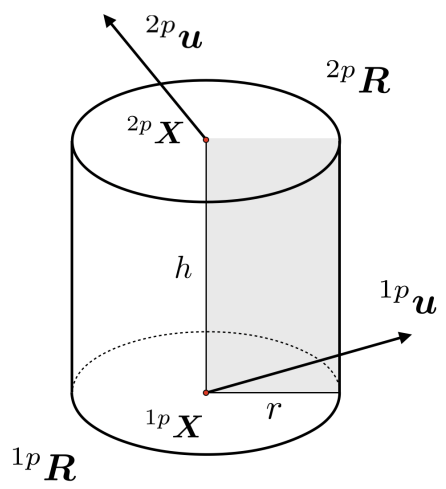

Figure 5: Kinematical descriptors adopted to describe the pivot.

Finally, in view of (17) and (18), we are allowed to choose the following deformation measures for the pivot,

$$
\begin{aligned}
{ }^{p} \boldsymbol{e} & =\overline{\boldsymbol{R}}^{\top}\left({ }^{2 p} \boldsymbol{u}-{ }^{1 p} \boldsymbol{u}\right)+\left(\overline{\boldsymbol{R}}^{\top}-\boldsymbol{I}\right)\left({ }^{2 p} \boldsymbol{X}-{ }^{1 p} \boldsymbol{X}\right) \\
{ }^{p} \boldsymbol{E} & =\overline{\boldsymbol{R}}^{\top}\left({ }^{2 p} \boldsymbol{R}-{ }^{1 p} \boldsymbol{R}\right)=2 \text { skew }\left[\left({ }^{1 p} \boldsymbol{R}^{\top}{ }^{2 p} \boldsymbol{R}\right)^{1 / 2}\right]
\end{aligned}
$$

where skew $\left[\left({ }^{1 p} \boldsymbol{R}^{\top}{ }^{2 p} \boldsymbol{R}\right)^{1 / 2}\right]$ denotes the skew part of $\left({ }^{1 p} \boldsymbol{R}^{\top}{ }^{2 p} \boldsymbol{R}\right)^{1 / 2}$ and $\overline{\boldsymbol{R}}$ is the Riemannian mean between the two rotations ${ }^{1 p} \boldsymbol{R}$ and ${ }^{2 p} \boldsymbol{R}$, defined as follows

$$
\overline{\boldsymbol{R}}={ }^{1 p} \boldsymbol{R}\left({ }^{1 p} \boldsymbol{R}^{\top}{ }^{2 p} \boldsymbol{R}\right)^{1 / 2}={ }^{2 p} \boldsymbol{R}\left({ }^{2 p} \boldsymbol{R}^{\top}{ }^{1 p} \boldsymbol{R}\right)^{1 / 2}
$$

It is worth noticing that the mean rotation satisfies the relationship

$$
{ }^{1 p} \boldsymbol{R}^{\top} \overline{\boldsymbol{R}}=\overline{\boldsymbol{R}}^{\top}{ }^{2 p} \boldsymbol{R}
$$

namely, the relative rotation between ${ }^{1 p} \boldsymbol{R}$ and $\overline{\boldsymbol{R}}$ is equal to that between $\overline{\boldsymbol{R}}$ and ${ }^{2 p} \boldsymbol{R}$. In other words, if the Riemannian metric is adopted, $\overline{\boldsymbol{R}}$ lies midway between ${ }^{1 p} \boldsymbol{R}$ and ${ }^{2 p} \boldsymbol{R}$ along the shortest geodesic curve connecting them in $S O(3)$ (Moakher, 2002).

Analogously to the case of fibers, the axial vector of the skew tensor ${ }^{p} \boldsymbol{E}$, i.e.,

$$
{ }^{p} \boldsymbol{\kappa}={ }^{p} \boldsymbol{E}_{\times}
$$

represents the deformation measures associated to the relative change of rotations between the two bases of the pivot.

To evaluate Eq. (20) and (21), we introduce the orthogonal tensor

$$
\boldsymbol{Q}=Q_{\alpha \beta} \boldsymbol{b}_{\alpha} \otimes \boldsymbol{b}_{\beta}
$$

that defines the basis transformation

$$
c_{\alpha}=Q_{\beta \alpha} b_{\beta}
$$

specifically, a counterclockwise rotation of $\pi / 2$ around the $\boldsymbol{b}_{3}$ axis

$$
\left(\begin{array}{lll}
\boldsymbol{c}_{1} & \boldsymbol{c}_{2} & \boldsymbol{c}_{3}
\end{array}\right)=\left(\begin{array}{ccc}
0 & -1 & 0 \\
1 & 0 & 0 \\
0 & 0 & 1
\end{array}\right)^{\top}\left(\begin{array}{lll}
\boldsymbol{b}_{1} & \boldsymbol{b}_{2} & \boldsymbol{b}_{3}
\end{array}\right) .
$$


Therefore, the relative rotation between the two bases of the pivot can be expressed by components in the basis $\left\{\boldsymbol{b}_{i}\right\}$ as

$$
{ }^{12} \boldsymbol{R}={ }^{1 p} \boldsymbol{R}^{\top}{ }^{2 p} \boldsymbol{R}={ }^{1 p} \boldsymbol{R}_{\alpha i} Q_{\alpha \gamma}{ }^{2 p} \tilde{\boldsymbol{R}}_{\gamma \beta} Q_{j \beta} \boldsymbol{b}_{i} \otimes \boldsymbol{b}_{j} .
$$

By using Rodrigues' formula, the relative rotation can be expressed as follows

$$
{ }^{12} \boldsymbol{R}=\boldsymbol{I}+\sin (\psi) \boldsymbol{K}+[1-\cos (\psi)] \boldsymbol{K}^{2}
$$

depending on a counterclockwise angle $\psi$, and the axis of rotation $\boldsymbol{k}=\boldsymbol{K}_{\times}$, where $\boldsymbol{K}$ is a skew tensor defined by

$$
{ }^{12} \boldsymbol{R}=\exp (\psi \boldsymbol{K}) \rightarrow \log \left({ }^{12} \boldsymbol{R}\right)=\psi \boldsymbol{K} .
$$

By knowing the matrix ${ }^{12} \boldsymbol{R}$, it is possible to determine the angle $\psi$ by the relationships

$$
\begin{aligned}
& \cos (\psi)=\frac{1}{2}\left[\operatorname{tr}\left({ }^{12} \boldsymbol{R}\right)-1\right], \\
& \sin (\psi)=\frac{1}{2} \sqrt{\left[3-\operatorname{tr}\left({ }^{12} \boldsymbol{R}\right)\right]\left[1+\operatorname{tr}\left({ }^{12} \boldsymbol{R}\right)\right]} .
\end{aligned}
$$

An efficient way to do this is to use the '2-argument arctangent' function, which returns a single value of $\psi$ such that $-\pi<\psi \leq \pi$. Moreover, the tensor $\boldsymbol{K}$ can be calculated by

$$
\boldsymbol{K}= \begin{cases}\mathbf{0}, & \text { for } \psi=0 \\ \frac{1}{2 \sin (\psi)}\left({ }^{12} \boldsymbol{R}-{ }^{12} \boldsymbol{R}^{\top}\right), & \text { for } \psi \neq 0 \text { and }|\psi|<\pi\end{cases}
$$

Finally, recalling that the square root of a rotation matrix is a rotation matrix with the same axis and a rotation angle which is half of that of the original matrix, we have

$$
\left({ }^{12} \boldsymbol{R}\right)^{1 / 2}=\boldsymbol{I}+\sin \left(\frac{\psi}{2}\right) \boldsymbol{K}+\left[1-\cos \left(\frac{\psi}{2}\right)\right] \boldsymbol{K}^{2} .
$$

By expressing the deformation measurements by components in the base $\left\{\boldsymbol{b}_{i}\right\}$, we obtain

$$
{ }^{p} \boldsymbol{e}=\left[\bar{R}_{j i}\left({ }^{2 p} u_{j}-{ }^{1 p} u_{j}\right)+\left(\bar{R}_{j i}-\delta_{i j}\right)\left({ }^{2 p} X_{j}-{ }^{1 p} X_{j}\right)\right] \boldsymbol{b}_{i}
$$

and

$$
{ }^{p} \boldsymbol{\kappa}={ }^{p} E_{32} \boldsymbol{b}_{1}+{ }^{p} E_{13} \boldsymbol{b}_{2}+{ }^{p} E_{21} \boldsymbol{b}_{3} .
$$

\section{Continuum modeling}

Being $\ell$ the distance between two neighboring pivots, the Representative Elementary Volume (REV) of our structure is assumed to have a square base of length $\ell$ centered in a pivot (see Fig. 6) and height $t$ equal to the thickness of the structure.

The specific geometry of the fiber lattice allows us to take the following identifications for granted

$$
\begin{aligned}
& { }^{1 p} \boldsymbol{X}={ }^{1} \boldsymbol{X}\left(s_{1}\right), \quad{ }^{2 p} \boldsymbol{X}={ }^{2} \boldsymbol{X}\left(s_{2}\right), \\
& { }^{1 p} \boldsymbol{u}\left({ }^{1 p} \boldsymbol{X}\right)={ }^{1} \boldsymbol{u}\left(s_{1}\right), \quad{ }^{2 p} \boldsymbol{u}\left({ }^{2 p} \boldsymbol{X}\right)={ }^{2} \boldsymbol{u}\left(s_{2}\right), \\
& { }^{1 p} \boldsymbol{R}={ }^{1} \boldsymbol{R}\left(s_{1}\right), \quad{ }^{2 p} \boldsymbol{R}={ }^{2} \boldsymbol{R}\left(s_{2}\right) .
\end{aligned}
$$

According to the previous definitions, we can easily interpret the components of the vector fields

$$
\begin{aligned}
& { }^{\alpha} \boldsymbol{e}=\left({ }^{\alpha} \varepsilon,{ }^{\alpha} \gamma_{p},{ }^{\alpha} \gamma_{n}\right), \quad{ }^{\alpha} \kappa=\left({ }^{\alpha} \kappa_{\tau},{ }^{\alpha} \kappa_{n},{ }^{\alpha} \kappa_{g}\right), \\
& { }^{p} \boldsymbol{e}=\left({ }^{p} \gamma_{1},{ }^{p} \gamma_{2},{ }^{p} \varepsilon\right), \quad{ }^{p} \boldsymbol{\kappa}=\left({ }^{p} \kappa_{1},{ }^{p} \kappa_{2},{ }^{p} \kappa_{\tau}\right),
\end{aligned}
$$




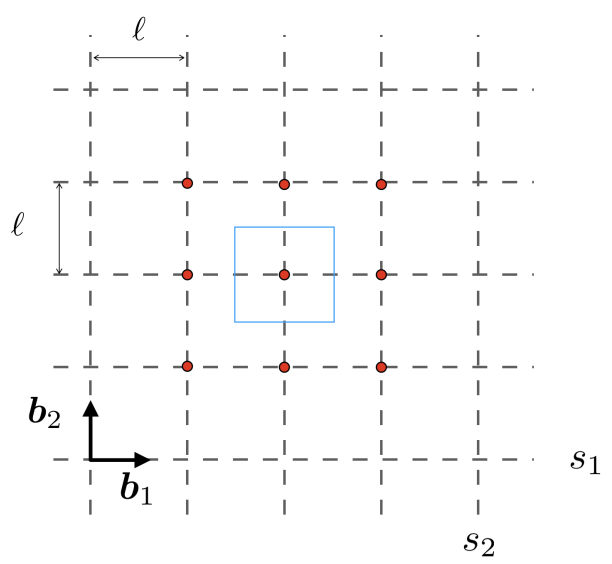

Figure 6: Beam lattice and unit reference cell for the homogenization (light blue square).

as measures of deformation for the fibers and the pivots that are continuously distributed over the considered domain. Specifically, they are: the elongations ${ }^{\alpha} \varepsilon$, the shears along the in-plane ${ }^{\alpha} \gamma_{p}$, and the out-of-plane direction ${ }^{\alpha} \gamma_{n}$, the torsions ${ }^{\alpha} \kappa_{\tau}$, and the normal ${ }^{\alpha} \kappa_{n}$, and geodesic ${ }^{\alpha} \kappa_{g}$ bending for the fibers; the shears along the in-plane directions ${ }^{p} \gamma_{1}$ and ${ }^{p} \gamma_{2}$, the elongation ${ }^{p} \varepsilon$, the bending along the in-plane directions ${ }^{p} \kappa_{1}$ and ${ }^{p} \kappa_{2}$, and the torsion ${ }^{p} \kappa_{\tau}$ for the connections due to the pivots.

We assume that the strain energy density is a quadratic form with the following two contributions

$$
\begin{aligned}
\pi_{f}\left({ }^{\alpha} \boldsymbol{e},{ }^{\alpha} \boldsymbol{\kappa}\right)=\frac{1}{2}\left[\mathscr{K}_{e}\left({ }^{1}{ }^{2}{ }^{2}+{ }^{2} \varepsilon^{2}\right)+\mathscr{K}_{\gamma_{p}}\left({ }^{1} \gamma_{p}{ }^{2}+{ }^{2} \gamma_{p}{ }^{2}\right)+\right. \\
\\
\mathscr{K}_{\gamma_{n}}\left({ }^{1} \gamma_{n}{ }^{2}+{ }^{2} \gamma_{n}{ }^{2}\right)+{ }^{f} \mathscr{K}_{\tau}\left({ }^{1} \kappa_{\tau}{ }^{2}+{ }^{2} \kappa_{\tau}{ }^{2}\right)+ \\
\left.\mathscr{K}_{n}\left({ }^{1} \kappa_{n}{ }^{2}+{ }^{2} \kappa_{n}{ }_{n}^{2}\right)+\mathscr{K}_{g}\left({ }^{1} \kappa_{g}{ }^{2}+{ }^{2} \kappa_{g}{ }^{2}\right)\right]
\end{aligned}
$$

and

$$
\begin{aligned}
\pi_{p}\left({ }^{p} \boldsymbol{e},{ }^{p} \boldsymbol{\kappa}\right)= & \frac{1}{2}\left[\mathscr{K}_{\gamma}\left({ }^{p} \gamma_{1}{ }^{2}+{ }^{p} \gamma_{2}{ }^{2}\right)+\mathscr{K}_{b}\left({ }^{p} \kappa_{1}{ }^{2}+{ }^{p} \kappa_{2}{ }^{2}\right)\right. \\
& \left.+{ }^{p} \mathscr{K}_{e}{ }^{p} \varepsilon^{2}+{ }^{p} \mathscr{K}_{\tau}{ }^{p} \kappa_{\tau}{ }^{2}\right],
\end{aligned}
$$

respectively, for the fibers and the pivots, when the limit for $\ell$ tending to zero is carried out. The material parameters ${ }^{f} \mathscr{K}_{e}, \mathscr{K}_{\gamma_{p}}, \mathscr{K}_{\gamma_{n}},{ }^{f} \mathscr{K}_{\tau}, \mathscr{K}_{n}$, and $\mathscr{K}_{g}$ are positive constants, assumed to be equal for the two embedded fibers, and represent the stiffnesses related to the elongation, in-plane and out-of-plane shear, twisting, normal and geodesic bending, respectively. Besides, ${ }^{p} \mathscr{K}_{e},{ }^{p} \mathscr{K}_{\tau}, \mathscr{K}_{\gamma}$ and $\mathscr{K}_{b}$ are the constitutive positive constants related to the elongation, torsion, shear and bending stiffnesses of the pivots. All these parameters can be evaluated as a first approximation with the formulae

$$
\begin{aligned}
f_{\mathscr{K}_{e}} & =\frac{\mathrm{E}^{f} A}{\ell}, \quad \mathscr{K}_{\gamma_{p}}=\frac{\mathrm{k}_{p} \mathrm{G}^{f} A}{\ell}, \quad \mathscr{K}_{\gamma_{n}}=\frac{\mathrm{k}_{n} \mathrm{G}^{f} A}{\ell}, \\
f_{\mathscr{K}_{\tau}} & =\frac{\mathrm{q} \mathrm{G}^{f} J_{\tau}}{\ell}, \quad \mathscr{K}_{n}=\frac{\mathrm{E} J_{n}}{\ell}, \quad \mathscr{K}_{g}=\frac{\mathrm{E} J_{g}}{\ell}, \\
p_{\mathscr{K}_{e}} & =\frac{\mathrm{E}^{p} A}{\ell^{2} h}, \quad \mathscr{K}_{\gamma}=\frac{12 E^{p} \boldsymbol{J}}{\ell^{2} h^{3}}, \\
{ }^{p} \mathscr{K}_{\tau} & =\frac{\mathrm{G}^{p} J_{\tau}}{\ell^{2} h}, \quad \mathscr{K}_{b}=\frac{4 \mathrm{E}^{p} \boldsymbol{J}}{\ell^{2} h},
\end{aligned}
$$

where $\mathrm{E}$ and $\mathrm{G}$ are the Young and shear moduli, respectively, ${ }^{f} A$ is the cross-section area, $J_{g}$ is the second moment of 
Table 1

Fiber and pivot dimensions in $\mathrm{mm}$.

\begin{tabular}{llllll}
\hline$b_{b}$ & $h_{b}$ & $r_{p}$ & $L$ & $l$ & $\ell$ \\
\hline 1.6 & 1.0 & 0.45 & 210 & 70 & 4.95 \\
\hline
\end{tabular}

\section{Table 2}

Material parameters of polyamide PA 2200.

\begin{tabular}{ll}
\hline Mechanical property & Value \\
\hline Mass density, $\varrho$ & $0.93 \mathrm{~g} \mathrm{~cm}^{-3}$ \\
\hline Young's modulus, $E$ & $1600 \mathrm{~N} \mathrm{~mm}^{-2}$ \\
\hline Poisson's ratio, $v$ & 0.4 \\
\hline
\end{tabular}

area for the geodesic bending, $J_{n}$ is the second moment of area for the out-of-plane bending, ${ }^{f} J_{\tau}$ is the polar moment of area, while $\mathrm{k}_{p}, \mathrm{k}_{n}, q$ are positive constants. Finally, ${ }^{p} A,{ }^{p} \boldsymbol{J}_{\tau}$, and ${ }^{p} \boldsymbol{J}$ are the area, the torsional, and the flexural second moment of area of the pivot, respectively. In this paper, we have proposed a possible choice for the deformation energy. Nonetheless, a more general form of energy also involving couplings (possibly non-quadratic) between the measures of deformation can be postulated (see, e.g., Rizzi and Tatone (1996)). In the light of the findings presented in (Auger et al., 2020), where a not negligible Poynting effect ${ }^{2}$ (Poynting, 1909) could be associated with the pivots, this kind of generalization may result in a better predictive ability of the macro model.

\section{Numerical simulations}

To test the proposed model, we perform some numerical simulations directly employing the weak formulation

$$
\delta \int_{B}\left(\pi_{f}+\pi_{p}\right) \mathrm{d} \Omega=0
$$

in a finite element code. Unless stated otherwise, the code solves the variational problem (45), making use of essential boundary conditions on displacements and rotations fields and void of external actions that exert a further work contribution. Table 1 lists the main geometrical characteristics of the specimen used in the tests, namely the sizes of the rectangular cross-section of the fibers, the width $b_{b}$ and the thickness $h_{b}$, the radius of the pivots $r_{p}$, the dimensions of the entire sample, $L$ and $l$, and the pitch between fibers, $\ell$. Table 2 reports the material characteristic of the sample assumed to be made of polyamide PA 2200.

As far as concern the height of the pivots, we consider three values, i.e., $1 \mathrm{~mm}, 2.5 \mathrm{~mm}, 5 \mathrm{~mm}$. Naturally, the more they are high, the more they are deformable. Thus, accurately describing their deformation becomes progressively essential. As it can be seen from Eq. (33), the reference configuration is characterized by an undetermined axis of rotation being the relative rotation ${ }^{12} \boldsymbol{R}$ equal to the identity. To overcome such an issue in the numerical code, we initialize the numerical analysis starting from a configuration slightly different from the reference one. The code, indeed, implements a damped Newton's method approach, which automatically updates the Jacobian.

A first testing taken into consideration is the common bias extension test. In Figs. 7, 8, and 9 are displayed the equilibrium shapes for the samples with $1 \mathrm{~mm}, 2.5 \mathrm{~mm}, 5 \mathrm{~mm}$ high pivots, respectively. The colors indicate the average value of the out-of-plane displacement, i.e.

$$
\frac{1}{2}\left[{ }^{1} u_{3}(\boldsymbol{X})+{ }^{2} u_{3}(\boldsymbol{X})\right]
$$

where $\boldsymbol{X}$ is the center of the surface of the sample in its reference configuration. These figures exhibit how the considered macro-model is able to catch the chiral effect of the structure. As a matter of fact, an extension produces, because of the offset between the beams, a macroscopic twist of the specimen. We find that this behavior, as can be expected, increases as the offset rises.

\footnotetext{
${ }^{2}$ Poynting effect is the lengthening of a wire (in our case a slender beam) when twisted.
} 
Also, we analyze the deformation energy stored into the specimen during the bias test, by plotting its overall value and the values relative to the pivots and the beams.

In Figs. 10, 11, and 12, for the sake of comparison, we plot the total energy, and the two main contributions of it (pivots and beams) for diverse models. In detail, we consider

1. the standard Cauchy model in non-linear regime (micro model);

2. the proposed model with average rotations (labeled with AR);

3. the model with (latent) internal microstructure characterized by the fact that the only possible deformation of pivots is the torsion around their axis, assumed normal to the surface of the sample (labeled with C) Giorgio et al. (2017);

4. and a model where the deformations of the pivots are all allowed but with the hypothesis that they are small (labeled with Rs) Giorgio et al. (2019).

From these figures, we see that all the considered models provide results that are compatible with each other by an energy point of view (globally). Still, the model with latent internal microstructure is clearly not able to catch the chiral effect because lacking offset. Besides, the model with small rotations has a limited range of applicability. It is worth noticing that the material stiffnesses employed in the calculations are just roughly evaluated by the formulae (44). A more accurate estimate of them requires the solution of an inverse problem of identification, as done in (Placidi et al., 2017; De Angelo et al., 2019a).

Secondly, a shear test is performed to investigate also the buckling and post-buckling behavior of the considered model. In this case, we apply some small forces in the out-of-plane direction as a defect to favor the rising of the buckled shape. In particular, Fig. 13 shows the deformed equilibrium shape for an imposed shear displacement of $7 \mathrm{~cm}$ along with the out-of-plane displacement through colors. The deformed shapes obtained by the micro-model, which is very detailed and numerically expensive, and by the macro model AR are reasonably close. Moreover, it is worth noticing that the two peaks of the buckled shape are not anti-symmetric as one could be expected. This lack of symmetry in the equilibrium configuration is not only confirmed with the micro-model, but there is also experimental evidence that corroborated it, as shown in (Ganzosch et al., 2018; Barchiesi et al., 2019b). A possible explanation of this feature could be the effect of the interaction of two o more buckled modes with a very close energy content. In future works, we will investigate this aspect more deeply.

A similar good match can be found for the energy contributions, see Fig. 14. To have some hints about the postbuckling behavior, we also plot the out-of-plane displacement in two probe-points near the peaks of the buckled shape to monitoring their trend varying the imposed displacement. In this case, the critical displacement predicted by the macro model is a little overvalued due to the coarse approximation of the stiffnesses. However, with the exception of the critical value, the global behavior is notably similar.

Finally, a torsion test is performed, twisting the short ends of the rectangular pantograph with respect to one another. Specifically, the displacement fields ${ }^{1} \boldsymbol{u}$ and ${ }^{2} \boldsymbol{u}$ are fixed on one of the constrained edges as well as the Bryant angles $\left\{{ }^{1} \varphi_{1},{ }^{1} \varphi_{2},{ }^{1} \varphi_{3}\right\}$ and $\left\{{ }^{2} \varphi_{1},{ }^{2} \varphi_{2},{ }^{2} \varphi_{3}\right\}$. At the opposite edge, instead, in order to avoid the elaborate evaluation of the Bryant angles, a narrow strip is added, and a rigid torsion is imposed via displacements (taking into account the offset due to the presence of the pivots) until the twist angle of $\pi / 2$ is reached (see Fig. 16). This edge is free to move in the longitudinal direction of the specimen. The results concerning the different contributions of the deformation energy are reported in Fig. 17, where a comparison between the micro model and the proposed macro model is exhibited. Fig. 18 gives evidence of an elongation due to torsion, that we can call a Poynting effect, characterizes the torsional behavior of the pantographic structure.

\section{Conclusions}

A 2D shell endowed with a microstructure characterized by 12 scalar parameters has been introduced in order to model the nonlinear mechanical behavior of a pantographic structure (Altenbach and Eremeyev, 2014; Eremeyev and Pietraszkiewicz, 2016). The parameters account for the displacements and rotations of the beams' sections on the two layers of the pantograph. Effective invariant kinematical deformation measures have been defined, taking into account the offset of the two layers and introducing averages of rotations in $\mathrm{SO}(3)$ Lie group. A nonlinear elastic deformation energy, that is quadratic in the components of the deformation measures, has been defined. A rectangular continuum initially flat and subjected to extensional, shear, and torsion tests has been considered. The boundary value problems have been numerically solved by means of the COMSOL multiphysics software. The results have been compared with 


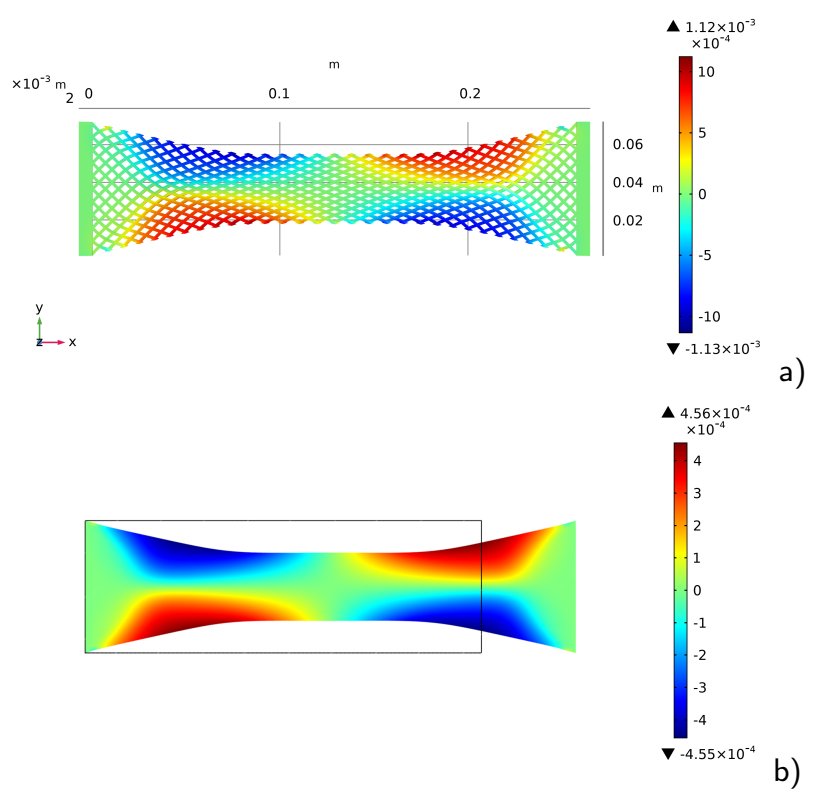

Figure 7: Pantographs with $1 \mathrm{~mm}$ high pivots under bias extension test: plot of the out-of-plane displacement for an imposed displacement of $5 \mathrm{~cm}$. Comparison between a) the 3D micro model and b) the proposed 2D macro model.

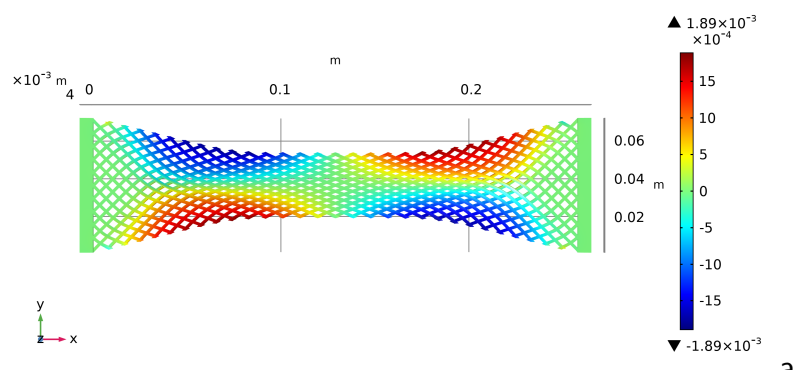

a)

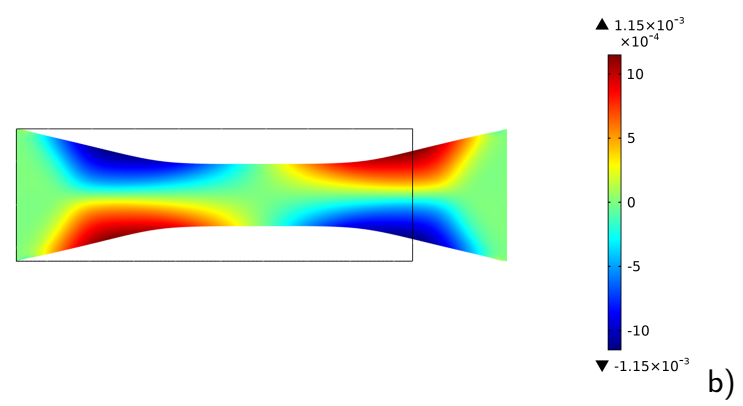

Figure 8: Pantographs with $2.5 \mathrm{~mm}$ high pivots under bias extension test: plot of the out-of-plane displacement for an imposed displacement of $5 \mathrm{~cm}$. Comparison between a) the 3D micro model and b) the proposed 2D macro model.

the ones obtained by using a model recently proposed by some of the authors and those obtained by modeling the pantograph with 3D nonlinear elastic FEs. The comparison has shown that the model proposed in the present paper is sufficiently effective. It is worth noting that both the extensional and shear tests show that the structure exhibits a buckled shape that, in the shear case, is not shew symmetric as one would expect. This behavior is confirmed by experimental tests (Ganzosch et al., 2018) and seems to be an effect of buckling modes interaction and deserves to be more carefully analyzed. In particular, more refined experimental tests need to be performed in order to confirm 


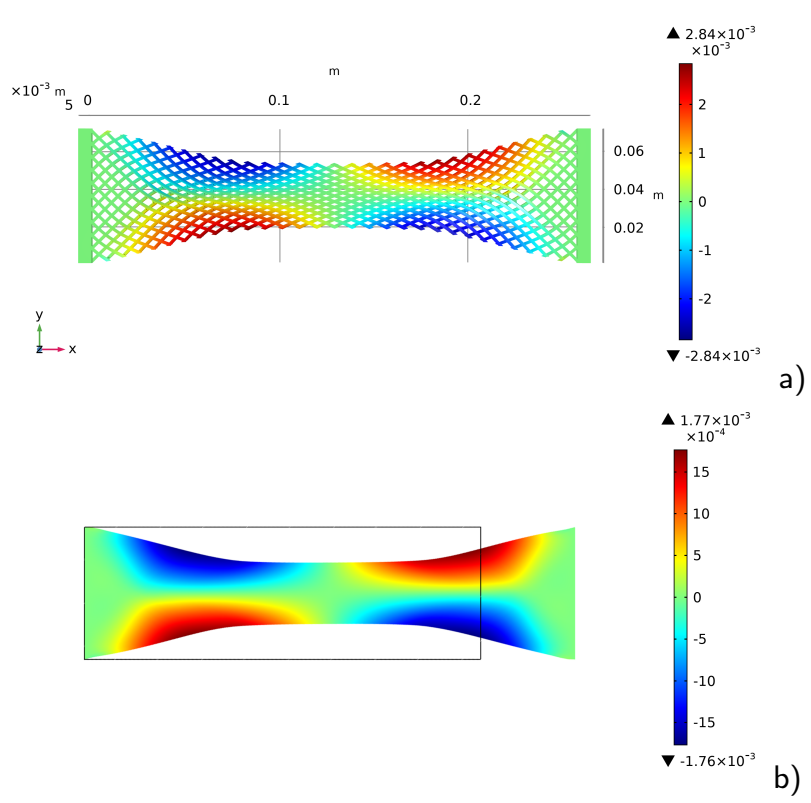

Figure 9: Pantographs with $5 \mathrm{~mm}$ high pivots under bias extension test: plot of the out-of-plane displacement for an imposed displacement of $5 \mathrm{~cm}$. Comparison between a) the 3D micro model and b) the proposed 2D macro model.

or revise the results obtained until now. Besides, the strain energy involving only quadratic terms of each one of the components of the deformation measures should be reconsidered in the light of some recent results that seem to show that the pivots can exhibit a non negligible Poynting effect. Finally, the torsion test shows that the proposed macro model is able to describe the Poynting effect characterizing the examined pantographic structure.

\section{References}

Abali, B.E., Wu, C.C., Müller, W.H., 2016. An energy-based method to determine material constants in nonlinear rheology with applications. Continuum Mechanics and Thermodynamics 28, 1221-1246.

Abdoul-Anziz, H., Seppecher, P., 2018. Strain gradient and generalized continua obtained by homogenizing frame lattices. Mathematics and mechanics of complex systems 6, 213-250.

Alibert, J.J., Seppecher, P., dell'Isola, F., 2003. Truss modular beams with deformation energy depending on higher displacement gradients. Mathematics and Mechanics of Solids 8, 51-73.

Altenbach, H., Eremeyev, V.A., 2014. Vibration analysis of non-linear 6-parameter prestressed shells. Meccanica 49, $1751-1761$.

Andreaus, U., Spagnuolo, M., Lekszycki, T., Eugster, S.R., 2018. A Ritz approach for the static analysis of planar pantographic structures modeled with nonlinear Euler-Bernoulli beams. Continuum Mechanics and Thermodynamics 30, 1103-1123.

Andres, J., Bersani, A.M., Leśniak, K., 2001. On some almost-periodicity problems in various metrics. Acta Applicandae Mathematica 65, 35-57.

Auger, P., Lavigne, T., Smaniotto, B., Spagnuolo, M., dell'Isola, F., Hild, F., 2020. Poynting effects in pantographic metamaterial captured via multiscale DVC. The Journal of Strain Analysis for Engineering Design , doi: 10.1177/0309324720976625.

Avella, M., Casale, L., Dell'Erba, R., Focher, B., Martuscelli, E., Marzetti, A., 1998. Broom fibers as reinforcing materials for polypropylene-based composites. Journal of Applied Polymer Science 68, 1077-1089.

Barchiesi, E., dell'Isola, F., Hild, F., Seppecher, P., 2020a. Two-dimensional continua capable of large elastic extension in two independent directions: asymptotic homogenization, numerical simulations and experimental evidence. Mechanics Research Communications $103,103466$.

Barchiesi, E., Eugster, S.R., dell'Isola, F., Hild, F., 2020b. Large in-plane elastic deformations of bi-pantographic fabrics: asymptotic homogenization and experimental validation. Mathematics and Mechanics of Solids 25, 739-767.

Barchiesi, E., Eugster, S.R., Placidi, L., dell'Isola, F., 2019a. Pantographic beam: A complete second gradient 1D-continuum in plane. Zeitschrift für angewandte Mathematik und Physik 70, 135.

Barchiesi, E., Ganzosch, G., Liebold, C., Placidi, L., Grygoruk, R., Müller, W.H., 2019b. Out-of-plane buckling of pantographic fabrics in displacement-controlled shear tests: experimental results and model validation. Continuum Mechanics and Thermodynamics $31,33-45$.

Barchiesi, E., Harsch, J., Ganzosch, G., Eugster, S.R., 2020c. Discrete versus homogenized continuum modeling in finite deformation bias extension test of bi-pantographic fabrics. Continuum Mechanics and Thermodynamics , 1-14.

Barchiesi, E., Placidi, L., 2017. A review on models for the 3D statics and 2D dynamics of pantographic fabrics, in: Wave dynamics and composite mechanics for microstructured materials and metamaterials. Springer, pp. 239-258.

Barchiesi, E., Spagnuolo, M., Placidi, L., 2019c. Mechanical metamaterials: a state of the art. Mathematics and Mechanics of Solids 24, $212-234$. 


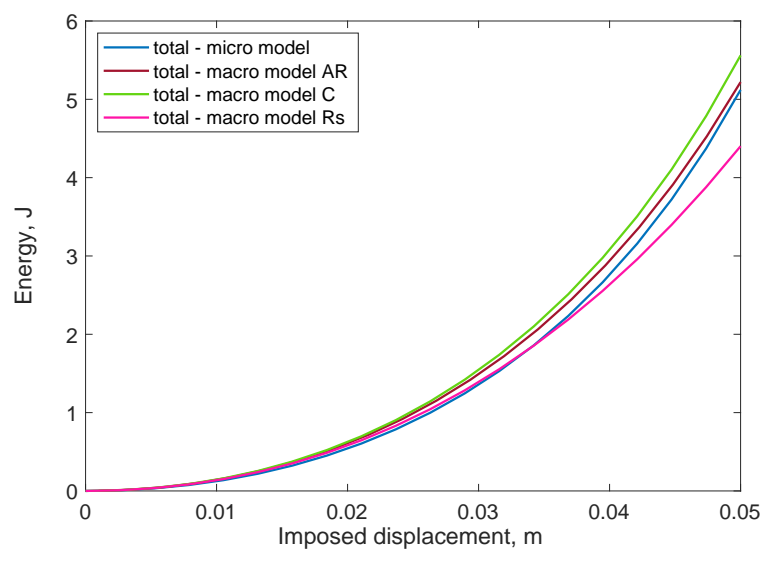

a)

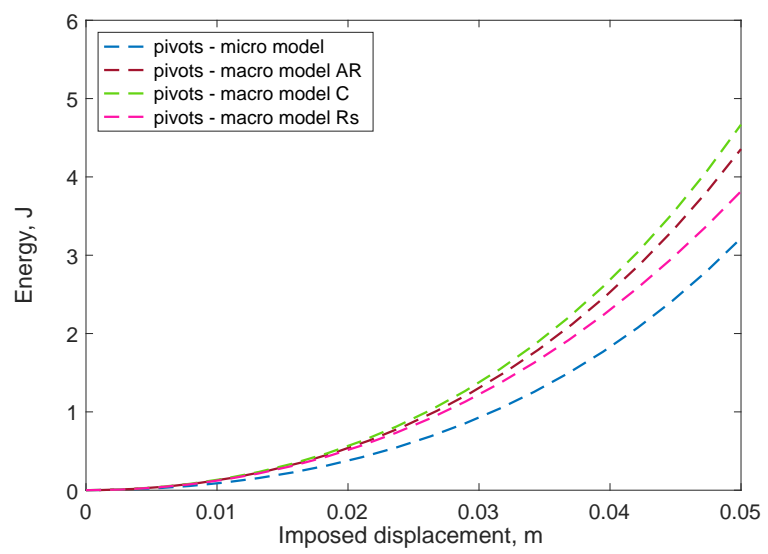

b)

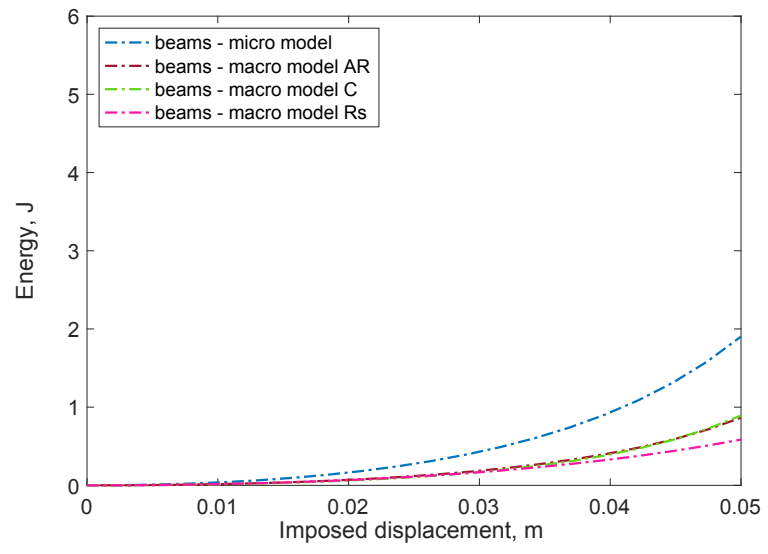

c)

Figure 10: Pantographs with $1 \mathrm{~mm}$ high pivots under bias extension test: stored energies for different models, a) total, b) pivot, c) beam contribution.

Berkache, K., Deogekar, S., Goda, I., Picu, R.C., Ganghoffer, J.F., 2019. Identification of equivalent couple-stress continuum models for planar random fibrous media. Continuum Mechanics and Thermodynamics 31, 1035-1050.

Bush, V., 1934. Structural analysis by electric circuit analogies. Journal of the Franklin Institute 217, 289-329.

Carter, G., Kron, G., 1944. Network analyzer solution of the equivalent circuits for elastic structures. Journal of the Franklin Institute 238, 443-452.

Ciallella, A., 2020. Research perspective on multiphysics and multiscale materials: a paradigmatic case. Continuum Mechanics and Thermodynamics 32, 527-539.

De Angelo, M., Barchiesi, E., Giorgio, I., Abali, B.E., 2019a. Numerical identification of constitutive parameters in reduced-order bi-dimensional models for pantographic structures: application to out-of-plane buckling. Archive of Applied Mechanics 89, 1333-1358.

De Angelo, M., Spagnuolo, M., D’Annibale, F., et al., 2019b. The macroscopic behavior of pantographic sheets depends mainly on their mi- 


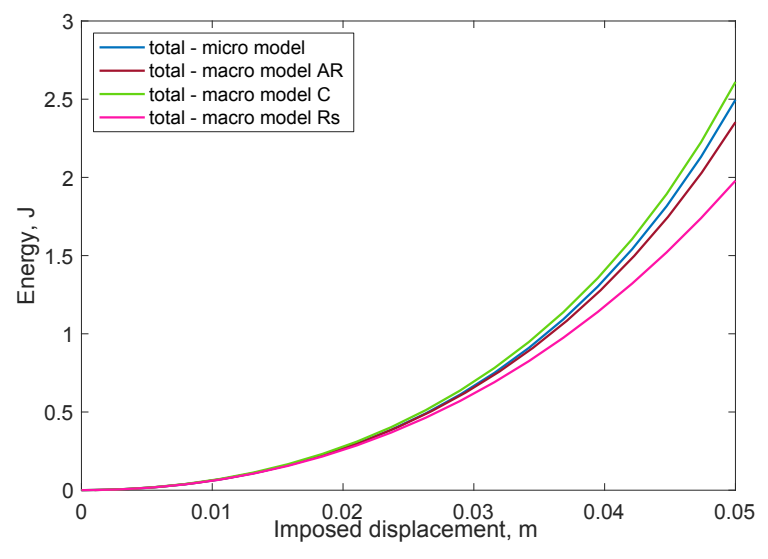

a)

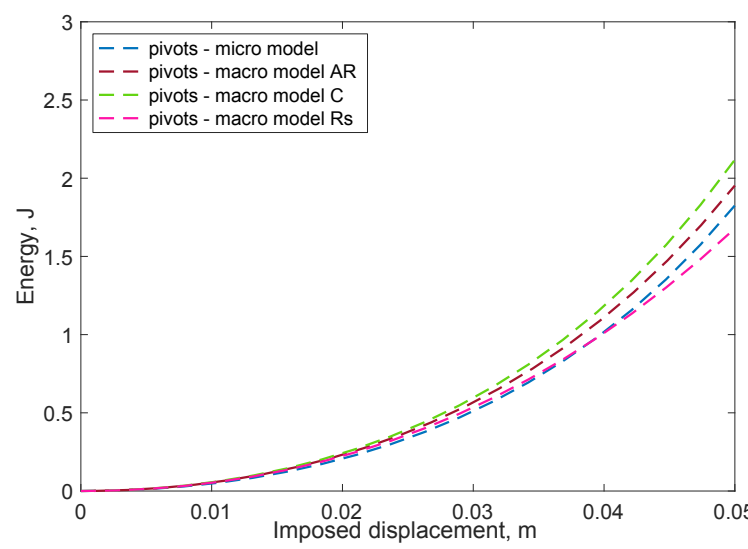

b)

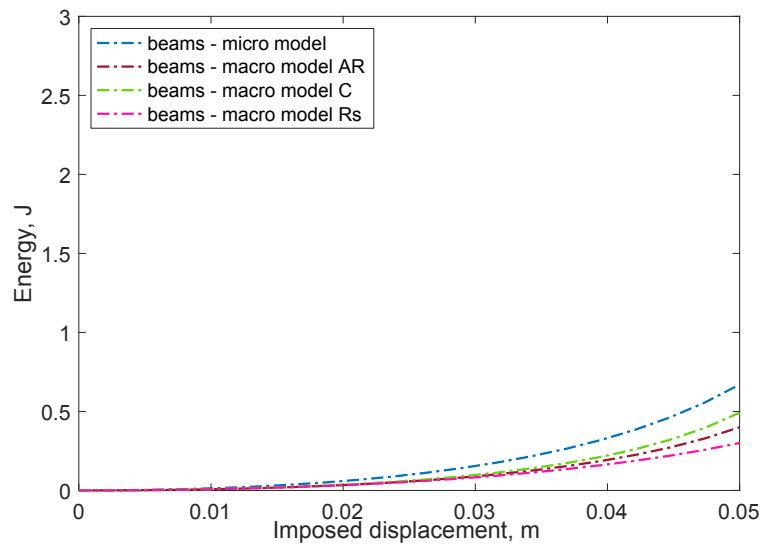

c)

Figure 11: Pantographs with $2.5 \mathrm{~mm}$ high pivots under bias extension test: stored energies for different models, a) total, b) pivot, c) beam contribution.

crostructure: experimental evidence and qualitative analysis of damage in metallic specimens. Continuum Mechanics and Thermodynamics 31 , $1181-1203$.

dell'Isola, F., Cuomo, M., Greco, L., Della Corte, A., 2017. Bias extension test for pantographic sheets: numerical simulations based on second gradient shear energies. Journal of Engineering Mathematics 103, 127-157.

dell'Isola, F., Lekszycki, T., Pawlikowski, M., Grygoruk, R., Greco, L., 2015. Designing a light fabric metamaterial being highly macroscopically tough under directional extension: first experimental evidence. Zeitschrift für angewandte Mathematik und Physik 66, 3473-3498.

dell'Isola, F., Maurini, C., Porfiri, M., 2004. Passive damping of beam vibrations through distributed electric networks and piezoelectric transducers: prototype design and experimental validation. Smart Materials and Structures 13, 299.

dell'Isola, F., Seppecher, P., Alibert, J.J., et al., 2019a. Pantographic metamaterials: an example of mathematically driven design and of its techno- 

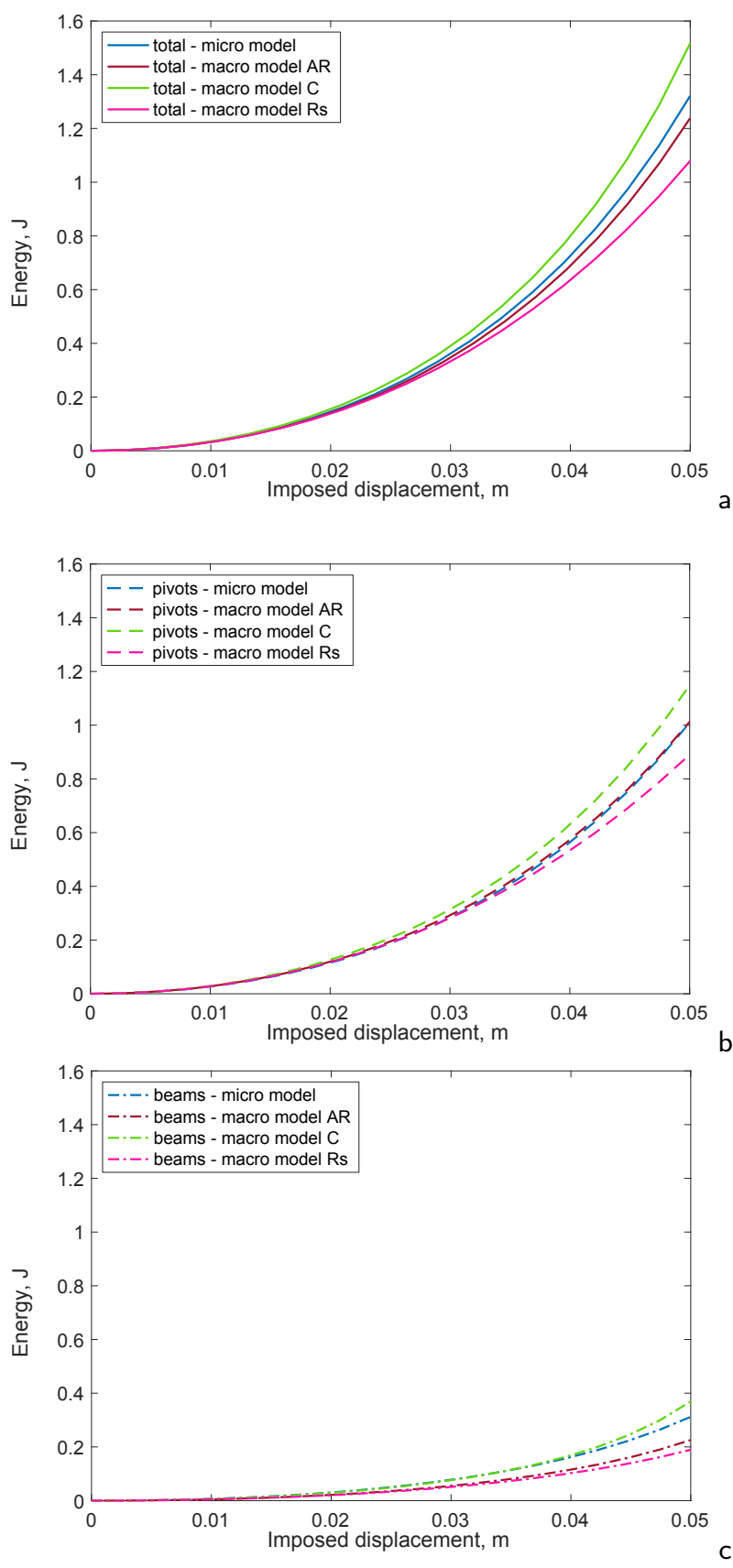

Figure 12: Pantographs with $5 \mathrm{~mm}$ high pivots under bias extension test: stored energies for different models, a) total, b) pivot, c) beam contribution.

logical challenges. Continuum Mechanics and Thermodynamics 31, 851-884.

dell'Isola, F., Seppecher, P., Spagnuolo, M., et al., 2019b. Advances in pantographic structures: design, manufacturing, models, experiments and image analyses. Continuum Mechanics and Thermodynamics 31, 1231-1282.

dell'Isola, F., Steigmann, D.J., 2020. Discrete and Continuum Models for Complex Metamaterials. Cambridge University Press.

dell'Isola, F., Turco, E., Misra, A., Vangelatos, Z., Grigoropoulos, C., Melissinaki, V., Farsari, M., 2019c. Force-displacement relationship in micro-metric pantographs: Experiments and numerical simulations. Comptes Rendus Mécanique 347, 397-405.

Eremeyev, V.A., 2019. Two-and three-dimensional elastic networks with rigid junctions: modeling within the theory of micropolar shells and solids. Acta Mechanica 230, 3875-3887.

Eremeyev, V.A., dell'Isola, F., 2020. Weak solutions within the gradient-incomplete strain-gradient elasticity. Lobachevskii Journal of Mathematics 


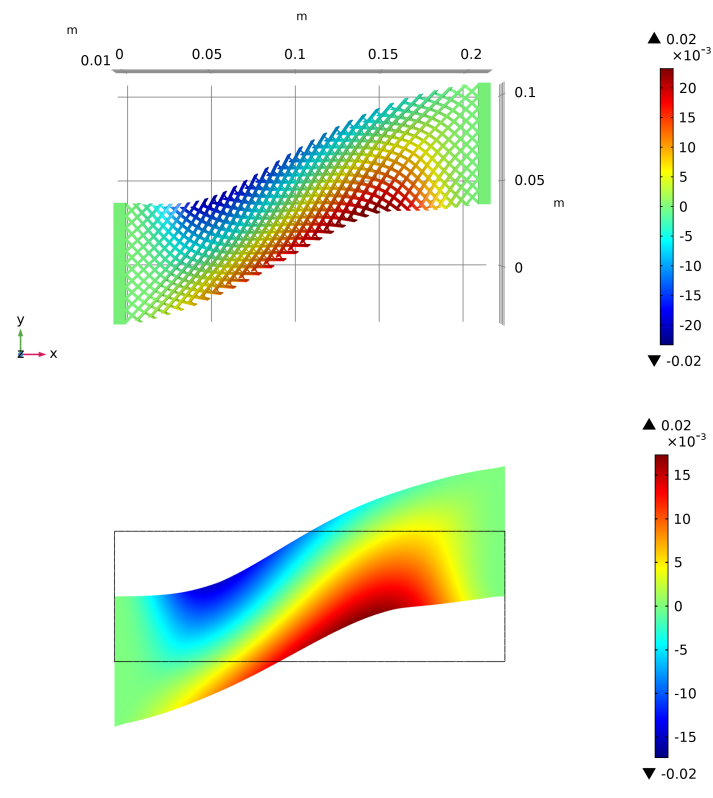

a)

b)

Figure 13: Pantographs with $5 \mathrm{~mm}$ high pivots under shear test: plot of the out-of-plane displacement for an imposed displacement of $7 \mathrm{~cm}$. Comparison between a) the 3D micro model and b) the proposed 2D macro model.

41, 191-197.

Eremeyev, V.A., dell'Isola, F., Boutin, C., Steigmann, D., 2018. Linear pantographic sheets: existence and uniqueness of weak solutions. Journal of Elasticity 132, 175-196.

Eremeyev, V.A., Pietraszkiewicz, W., 2016. Material symmetry group and constitutive equations of micropolar anisotropic elastic solids. Mathematics and Mechanics of Solids 21, 210-221.

Eremeyev, V.A., Turco, E., 2020. Enriched buckling for beam-lattice metamaterials. Mechanics Research Communications $103,103458$.

Ganzosch, G., Hoschke, K., Lekszycki, T., Giorgio, I., Turco, E., Müller, W., 2018. 3D-measurements of 3D-deformations of pantographic structures. Technische Mechanik 38, 233-245.

Germain, P., 2020. The method of virtual power in the mechanics of continuous media, I: Second-gradient theory. Mathematics and Mechanics of Complex Systems 8, 153-190.

Giorgio, I., Galantucci, L., Della Corte, A., Del Vescovo, D., 2015. Piezo-electromechanical smart materials with distributed arrays of piezoelectric transducers: current and upcoming applications. International Journal of Applied Electromagnetics and Mechanics 47, 1051-1084.

Giorgio, I., Rizzi, N.L., Andreaus, U., Steigmann, D.J., 2019. A two-dimensional continuum model of pantographic sheets moving in a 3D space and accounting for the offset and relative rotations of the fibers. Mathematics and Mechanics of Complex Systems 7, 311-325.

Giorgio, I., Rizzi, N.L., Turco, E., 2017. Continuum modelling of pantographic sheets for out-of-plane bifurcation and vibrational analysis. Proceedings of the Royal Society A: Mathematical, Physical and Engineering Sciences 473, 20170636.

Golaszewski, M., Grygoruk, R., Giorgio, I., Laudato, M., Di Cosmo, F., 2019. Metamaterials with relative displacements in their microstructure: technological challenges in 3d printing, experiments and numerical predictions. Continuum Mechanics and Thermodynamics 31, $1015-1034$.

Greco, L., 2020. An iso-parametric $G^{1}$-conforming finite element for the nonlinear analysis of Kirchhoff rod. Part I: the 2D case. Continuum Mechanics and Thermodynamics 32, 1473-1496.

Harrison, P., Alvarez, M.F., Anderson, D., 2018. Towards comprehensive characterisation and modelling of the forming and wrinkling mechanics of engineering fabrics. International Journal of Solids and Structures 154, 2-18.

Kron, G., 1945. Numerical solution of ordinary and partial differential equations by means of equivalent circuits. Journal of Applied Physics 16 , 172-186.

Laudato, M., Di Cosmo, F., Drobnicki, R., Göransson, P., 2019. Dynamical vector fields on pantographic sheet: Experimental observations, in: New Achievements in Continuum Mechanics and Thermodynamics. Springer, pp. 257-269.

Lossouarn, B., Deü, J.F., Aucejo, M., Cunefare, K.A., 2016. Multimodal vibration damping of a plate by piezoelectric coupling to its analogous electrical network. Smart Materials and Structures 25, 115042.

MacNeal, R.H., McCann, G.D., Wilts, C.H., 1951. The solution of aeroelastic problems by means of electrical analogies. Journal of the Aeronautical Sciences 18, 777-789.

Milton, G., Briane, M., Harutyunyan, D., 2017. On the possible effective elasticity tensors of 2-dimensional and 3-dimensional printed materials. Mathematics and Mechanics of Complex Systems 5, 41-94.

Milton, G.W., 2013. Complete characterization of the macroscopic deformations of periodic unimode metamaterials of rigid bars and pivots. Journal of the Mechanics and Physics of Solids 61, 1543-1560.

Milton, G.W., Cherkaev, A.V., 1995. Which elasticity tensors are realizable? J. Eng. Mater. Technol. 117, 483-493. 

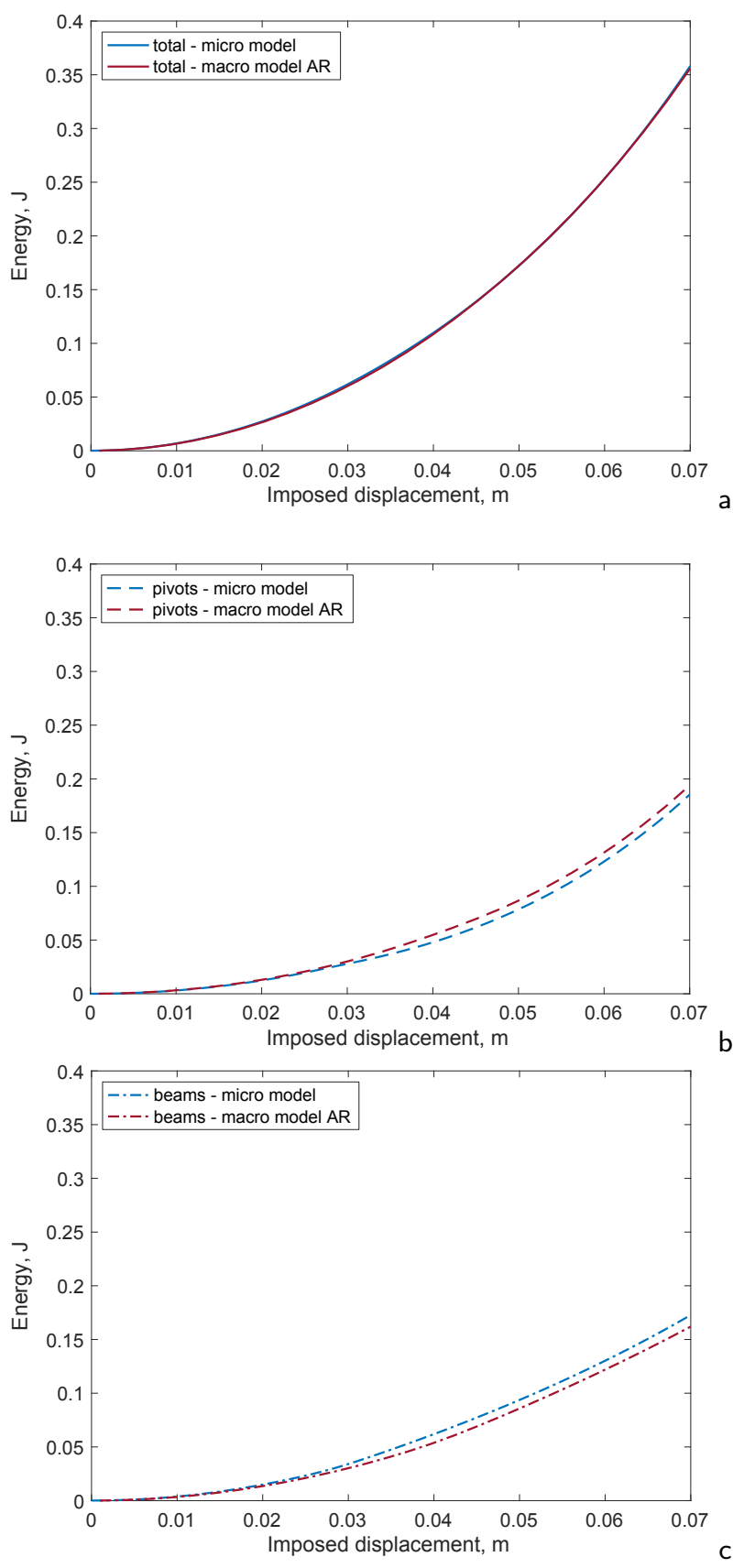

b)

c)

Figure 14: Pantographs with $5 \mathrm{~mm}$ high pivots under shear test: stored energies, a) total, b) pivot, c) beam contribution.

Misra, A., Poorsolhjouy, P., 2015. Identification of higher-order elastic constants for grain assemblies based upon granular micromechanics. Mathematics and Mechanics of Complex Systems 3, 285-308.

Moakher, M., 2002. Means and averaging in the group of rotations. SIAM journal on matrix analysis and applications 24, 1-16.

Nejadsadeghi, N., De Angelo, M., Drobnicki, R., Lekszycki, T., dell'Isola, F., Misra, A., 2019. Parametric experimentation on pantographic unit cells reveals local extremum configuration. Experimental Mechanics 59, 927-939.

Niiranen, J., Balobanov, V., Kiendl, J., Hosseini, S.B., 2019. Variational formulations, model comparisons and numerical methods for EulerBernoulli micro- and nano-beam models. Mathematics and Mechanics of Solids 24, 312-335.

Pideri, C., Seppecher, P., 1997. A second gradient material resulting from the homogenization of an heterogeneous linear elastic medium. Continuum Mechanics and Thermodynamics 9, 241-257.

Placidi, L., Andreaus, U., Della Corte, A., Lekszycki, T., 2015. Gedanken experiments for the determination of two-dimensional linear second 


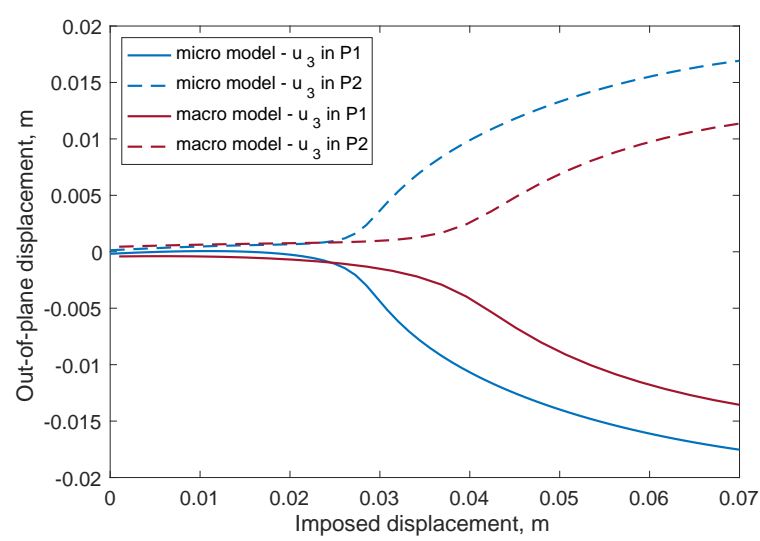

Figure 15: Pantographs with $5 \mathrm{~mm}$ high pivots under shear test. Out-of-plane displacement in probe points $P 1$ and $P 2$

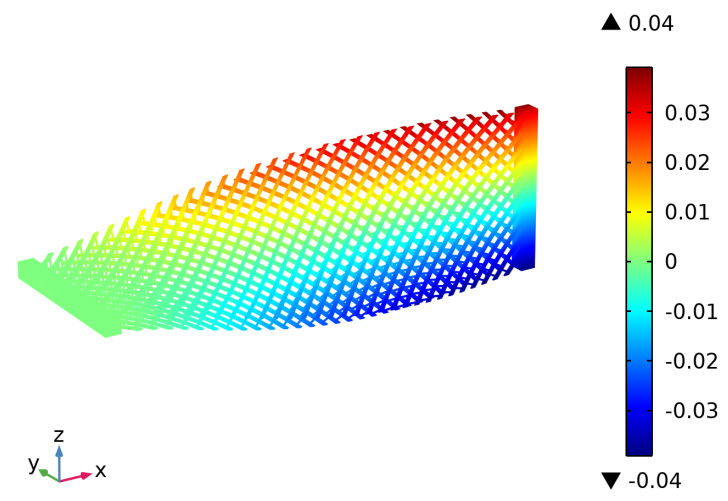

a)

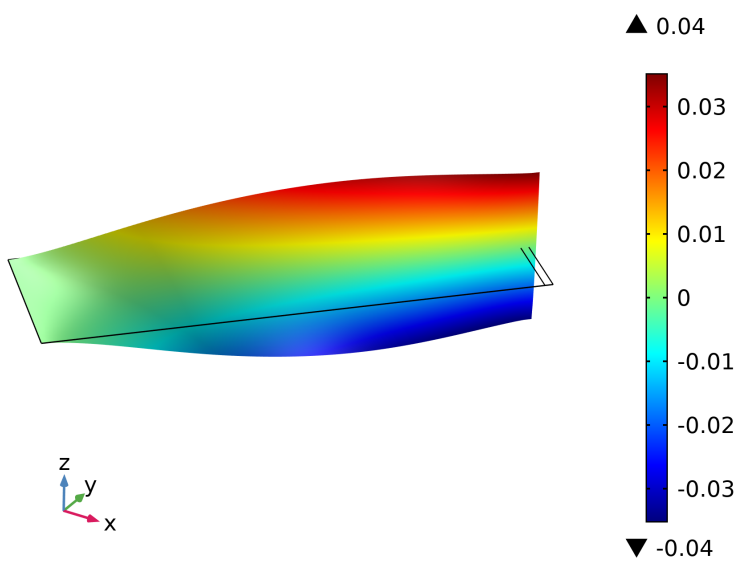

b)

Figure 16: Pantographs with $5 \mathrm{~mm}$ high pivots under torsion test: plot of the out-of-plane displacement for an imposed torsion of $\pi / 2$ rad. Comparison between a) the 3D micro model and b) the proposed 2D macro model. 

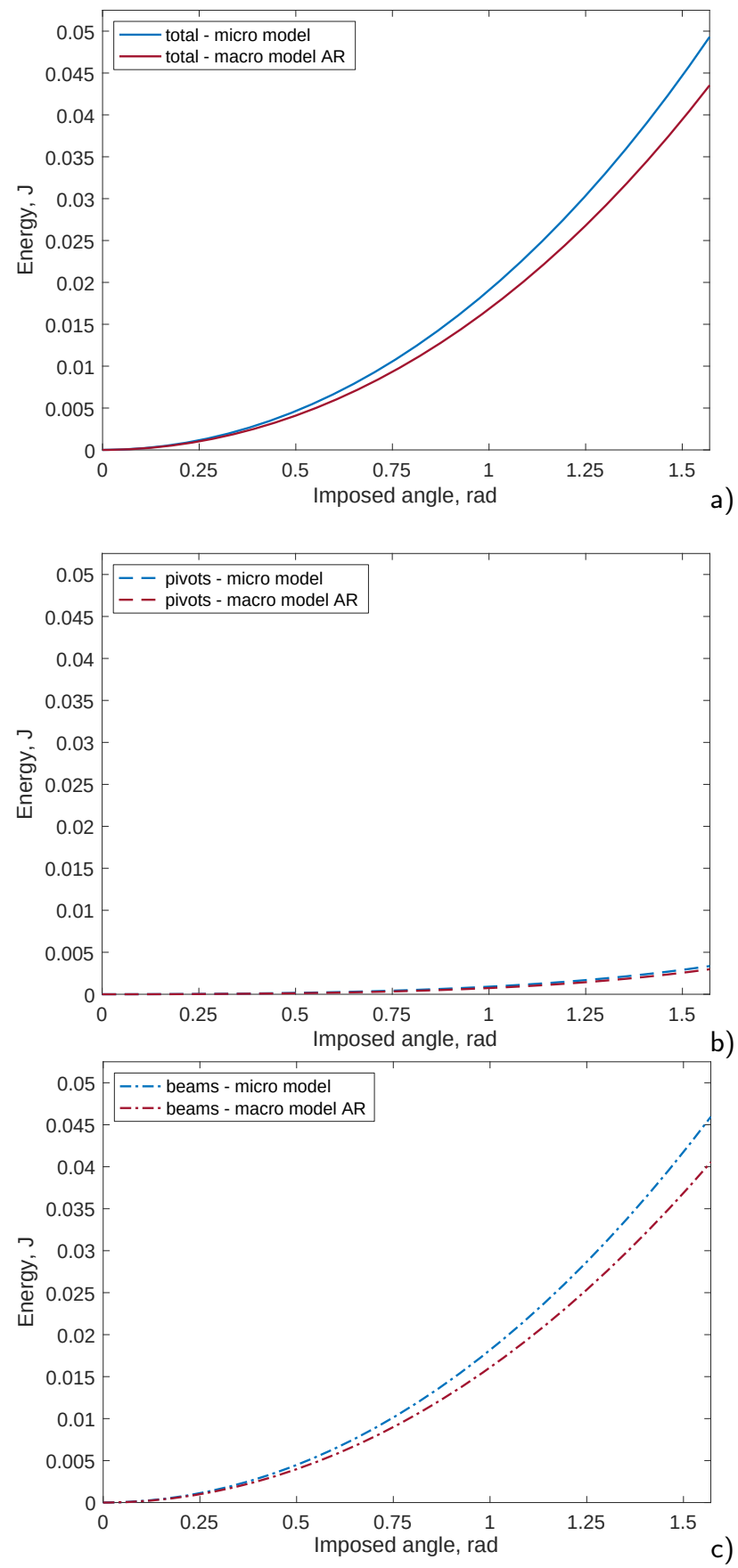

Figure 17: Pantographs with $5 \mathrm{~mm}$ high pivots under torsion test: stored energies, a) total, b) pivot, c) beam contribution.

gradient elasticity coefficients. Zeitschrift für angewandte Mathematik und Physik 66, 3699-3725.

Placidi, L., Andreaus, U., Giorgio, I., 2017. Identification of two-dimensional pantographic structure via a linear D4 orthotropic second gradient elastic model. Journal of Engineering Mathematics 103, 1-21.

Placidi, L., Barchiesi, E., Turco, E., Rizzi, N.L., 2016. A review on 2D models for the description of pantographic fabrics. Zeitschrift für angewandte Mathematik und Physik 67, 121.

Poynting, J.H., 1909. On pressure perpendicular to the shear planes in finite pure shears, and on the lengthening of loaded wires when twisted. Proceedings of the Royal Society of London. Series A, Containing Papers of a Mathematical and Physical Character 82, 546-559.

Rahali, Y., Eremeyev, V., Ganghoffer, J.F., 2020. Surface effects of network materials based on strain gradient homogenized media. Mathematics 


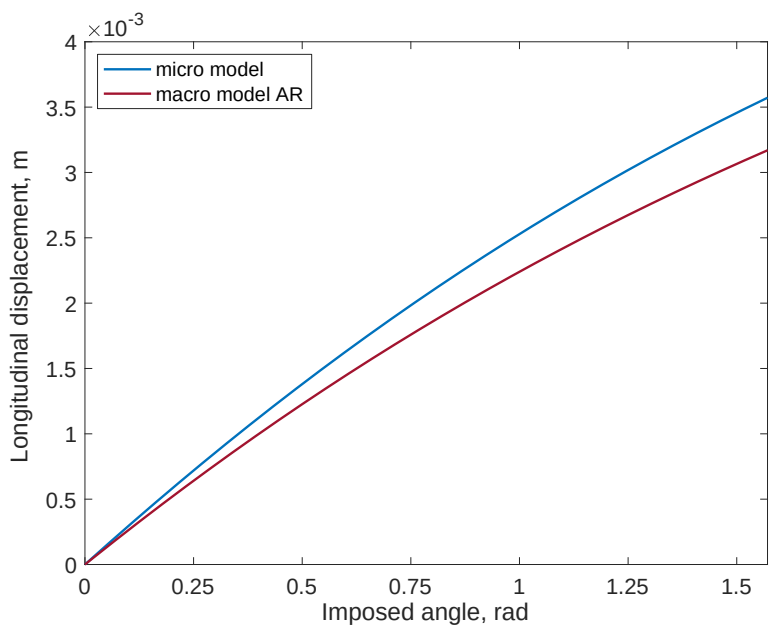

Figure 18: Pantographs with $5 \mathrm{~mm}$ high pivots under torsion test. Longitudinal translation of the short edge due to the Poynting effect.

and Mechanics of Solids 25, 389-406.

Rahali, Y., Goda, I., Ganghoffer, J.F., 2016. Numerical identification of classical and nonclassical moduli of 3D woven textiles and analysis of scale effects. Composite Structures 135, 122-139.

Rizzi, N., Tatone, A., 1996. Nonstandard models for thin-walled beams with a view to applications. Journal of Applied Mechanics 63, $399-403$.

Rosi, G., Placidi, L., Auffray, N., 2018. On the validity range of strain-gradient elasticity: a mixed static-dynamic identification procedure. European Journal of Mechanics-A/Solids 69, 179-191.

Seppecher, P., Alibert, J.J., dell'Isola, F., 2011. Linear elastic trusses leading to continua with exotic mechanical interactions, in: Journal of Physics: Conference Series, IOP Publishing. p. 012018.

Shirani, M., Luo, C., Steigmann, D.J., 2019. Cosserat elasticity of lattice shells with kinematically independent flexure and twist. Continuum Mechanics and Thermodynamics 31, 1087-1097.

Spagnuolo, M., Peyre, P., Dupuy, C., 2019. Phenomenological aspects of quasi-perfect pivots in metallic pantographic structures. Mechanics Research Communications 101, 103415.

Spagnuolo, M., Scerrato, D., 2020. The mechanical diode: On the tracks of James Maxwell employing mechanical-electrical analogies in the design of metamaterials, in: Developments and Novel Approaches in Biomechanics and Metamaterials. Springer, pp. 459-469.

Spagnuolo, M., Yildizdag, M.E., Andreaus, U., Cazzani, A.M., 2020. Are higher-gradient models also capable of predicting mechanical behavior in the case of wide-knit pantographic structures? Mathematics and Mechanics of Solids doi: 1081286520937339.

Steigmann, D., dell'Isola, F., Della Corte, A., 2015. Synthesis of fibrous complex structures: designing microstructure to deliver targeted macroscale response. Applied Mechanics Reviews 67.

Steigmann, D.J., dell'Isola, F., 2015. Mechanical response of fabric sheets to three-dimensional bending, twisting, and stretching. Acta Mechanica Sinica 31, 373-382.

Tran, C.A., Gołaszewski, M., Barchiesi, E., 2020. Symmetric-in-plane compression of polyamide pantographic fabrics—modelling, experiments and numerical exploration. Symmetry 12, 693.

Turco, E., 2019. How the properties of pantographic elementary lattices determine the properties of pantographic metamaterials, in: New achievements in continuum mechanics and thermodynamics. Springer, pp. 489-506.

Turco, E., Barchiesi, E., 2019. Equilibrium paths of Hencky pantographic beams in a three-point bending problem. Mathematics and Mechanics of Complex Systems 7, 287-310.

Turco, E., Golaszewski, M., Cazzani, A., Rizzi, N.L., 2016. Large deformations induced in planar pantographic sheets by loads applied on fibers: experimental validation of a discrete Lagrangian model. Mechanics Research Communications 76, 51-56.

Turco, E., Misra, A., Pawlikowski, M., dell'Isola, F., Hild, F., 2018. Enhanced Piola-Hencky discrete models for pantographic sheets with pivots without deformation energy: numerics and experiments. International Journal of Solids and Structures 147, 94-109.

Turco, E., Misra, A., Sarikaya, R., Lekszycki, T., 2019. Quantitative analysis of deformation mechanisms in pantographic substructures: experiments and modeling. Continuum Mechanics and Thermodynamics 31, 209-223.

Vasquez, F.G., Milton, G.W., Onofrei, D., 2011. Complete characterization and synthesis of the response function of elastodynamic networks. Journal of Elasticity 102, 31-54.

Yang, H., Abali, B.E., Timofeev, D., Müller, W.H., 2019. Determination of metamaterial parameters by means of a homogenization approach based on asymptotic analysis. Continuum Mechanics and Thermodynamics , 1-20.

Yildizdag, M.E., Tran, C.A., Barchiesi, E., Spagnuolo, M., dell'Isola, F., Hild, F., 2019. A multi-disciplinary approach for mechanical metamaterial synthesis: A hierarchical modular multiscale cellular structure paradigm, in: State of the Art and Future Trends in Material Modeling. Springer, pp. 485-505. 